\title{
Uber den klinischen Wert der Bestimmung des anorganischen Phosphors im Harne unnatürlich ernährter Säuglinge.
}

\section{(Berieht über 50 Fälle.)}

\author{
Von \\ Dr. Gisa Kaminer und Dr. Ernst Mayerhofer.
}

(Aus der k. k. Universitäts-Kinderklinik in Wien [Vorstand: Prof. Dr. Cl. Freih. v. Pirquet] und der Kinderabteilung des k. k. Kaiser-Franz-Josef-Spitales in Wien [Vorstand: Primarius Dozent Dr. Paul Moser].)

Mit 6 Textfiguren.

(Eingegangen am 27. März 1913.)

Nach der Mitteilung Molls ${ }^{1}$ ), daß im Harne des gesunden Brustkindes kein anorganisch gebundener Phosphor oder nur geringe Spuren davon ausgeschieden werden, gewann die Phosphatbestimmung im Harne der Säuglinge wieder Interesse und praktische Bedeutung. Die praktische Bedeutung des Mollschen Befundes liegt unserer Meinung nach darin, daß die Phosphatzahl beim natürlich genährten Säugling einen Harnbefund liefert, der ,gewissermaßen ein Spiegelbild des Ernährungszustandes des Kindes ist". Für den klinischen Betrieb ist es nun sehr wichtig, mit der Schnelligkeit einer Titration einen zur Beurteilung des Ernährungszustandes wichtigen Harnbefund zu erlangen; als erste bestätigende Mitteilung über die Richtigkeit des Mollschen Kriteriums für die Gesundheit eines Brustkindes brachten Mayerhofer und Pìibram²) den Befund, daß auch die mit konservierter Frauenmilch ernährten gesunden oder genesenden Säuglinge eine sehr kleine Ausscheidung anorganischen Phosphors im Harne aufweisen. Durch weiter ausgedehnte Untersuchungen stellte Mayerhofe $\mathrm{r}^{3}$ ) fest, daß bei beginnenden und vorgeschrittenen Verdauungs-

1) L. Moll, Die klinische Bedeutung der Phosphorausscheidung im Harn beim Brustkinde. Jahrb. f. Kinderheilk. 69, N. F. 19.

$\left.{ }^{2}\right)$ E. Mayerhofer u. E. Přibram, Ernährungsversuche bei Neugeborenen mit konservierter Frauenmilch. Wiener klin. Wochenschr. 1909, Nr. 26.

3) E. Mayerhofer, Chemische Teilerscheinungen im Harne gesunder und kranker Säuglinge. Zeitschr. f. Kinderheilk. 1, 487. 1911. 
störungen der Brustkinder gleichzeitig auch die organischen Bestandteile im Harne vermehrt sind, daß also auch die Permanganatzahi (Reduktionsindex) das gleiche Verhalten zeigt wie die Mollsche Phosphatzahl.

Die genannten Arbeiten beziehen sich größtenteils nur auf das Brustkind. Es wäre von großer praktischer Bedeutung, für das unnatürlich genährte Kind ăhnliche Regelmäßigkeiten zu finden; von diesem Gesichtspunkte aus haben wir diese klinischen Phosphatuntersuchungen auch auf den künstlich genährten Säugling ausgedehnt ${ }^{1}$ ).

Da wir zuerst den klinischen Wert der regelmäßig bei allen Kindern ausgeführten Phosphatbestimmungen erkennen wollten, so verzichteten wir auf eigentliche Stoffwechselversuche, sondern bestimmten nach dem Vorgange Molls die Phosphatmenge in je $10 \mathrm{ccm}$ beliebiger Harnportionen. Aus diesen ganz empirischen Massenuntersuchungen gewannen wir zuerst die Erkenntnis, daß das Kuhmilchkind bei einer Ernährungsstörung ein sehr starkes Ansteigen der anorganischen Harnphosphate aufweist, z. B.

*Fall 1. Ferdinand Wagmann, 5 Wochen alt; 4 Wochen Brust, seit 1 Woche bei 3stündlicher Darreichung von Viertelmilch Abmagerung, grüne, bröcklige Stühle, Erbrechen nach jeder Mahlzeit.

21. II. Aufnahme; 24stündige Teediät, schleimiger, dyspeptischer Stuhl; bei Tee kein Erbrechen.

22. II. Körpergewicht $2950 \mathrm{~g}$; kleines Kind, ausgetragen, ncch in gutem Ernährungszustand; lebhaft schreiend, normale Temperatur, kein Meteorismus; erhält heute 6 mal 100 Kuhmilch $1: 2$.

23. II. Körpergewicht $2970 \mathrm{~g}$; kein Erbrechen, doch 2 schleimige, dyspeptische Stühle. In $10 \mathrm{ccm} \mathrm{Harn} 6 \mathrm{mg} \mathrm{P}_{2} \mathrm{O}_{5}$.

2. III. Körpèrgewicht $3180 \mathrm{~g}$; bisher eine tägliche regelmäßige Gewichtszunahme von $28,8 \mathrm{~g}$. Die Stühle sind homogen geworden. In $10 \mathrm{ccm} \mathrm{Harn}$ $5 \mathrm{mg} \mathrm{P}_{2} \mathrm{O}_{5}$.

8. III. Körpergewicht $3200 \mathrm{~g}$; nach einem einmaligen Abfall von $50 \mathrm{~g}$ wieder langsame Zunahme; die Stühle bessern sich weiter. In $10 \mathrm{ccm} \mathrm{Harn} 5 \mathrm{mg} \mathrm{P}_{2} \mathrm{O}_{5}$.

11. III. Körpergewicht $3280 \mathrm{~g}$; bei homogenen Stühlen regelmäßige Gewichtszunahmen.

12. III. Körpergewicht $3300 \mathrm{~g}$; das Kind hat von gestern auf heute wieder schleimige, unverdaute Stühle.

13. III. Körpergewicht $3310 \mathrm{~g} ; 4$ schleimige, unverdaute Stühle. In $10 \mathrm{ccm}$ $\mathrm{Harn} 12 \mathrm{mg} \mathrm{P}_{2} \mathrm{O}_{5}$.

14. III. Körpergewicht $3350 \mathrm{~g}$; bei dyspeptischen Stühlen Erbrechen.

1) Die Untersuchungen erstrecken sich auf 4 Jahre; sie wurden teils am Materiale der Klinik, teils am Materiale der Moserschen Kinderabteilung ausgeführt; die mit * bezeichneten Fälle wurden bei Moser beobachtet. 
Resumee: Ein bei Drittelkuhmilch genesener und gut gedeihender Säugling (durchschnittliche Tageszunahme von $20 \mathrm{~g}$ während 17 Tagen), hat in je $10 \mathrm{ccm} \mathrm{Harn} 5-6 \mathrm{mg} \mathrm{P}_{2} \mathrm{O}_{5}$; anläßlich einer neuer lichen Verdaungsstörung steigen die Phosphate auf das Doppelte an $(12 \mathrm{mg}$ in $10 \mathrm{ccm}$ ).

Daß auch bei geringen und rasch vorübergehenden Verdauungsstörungen die Phosphatmengen steigen, beweist folgender Fall:

* Fall 2. Otto Knecht1, 4\% Monate alt. Das Kind erhielt die ersten 10 Tage Brust. Dann 2stündlich Kufekewasser (5 Kaffeelöffel Mehl auf $1 / 21$ Wasser). Daraufhin immer Verstopfung. Durch die Verabreichung von Kindermet und Kamillentee stellte sich eine leichte Besserung der Obstipation ein. Das Kind erhielt weiter Nestlemehl, doch ohne Erfolg. Seit 6 Wochen bekommt es sterilisierte Milch allein, nimmt aber auch nicht zu. 3-4topfige Stühle im Tage, leichter Husten, große Unruhe.

4. I. 1910. Aufnahme, 24 stündige Teediät.

5. I. 1910. Körpergewicht $3680 \mathrm{~g}$. Ein ausgetragenes Kind, seinem Alter entsprechend groß, stark abgenagert. Bis erbsengroße Lymphdrüsen beiderseits am Halse, in der Axilla und in der Leistengegend. Starke Spasmen der Extremitäten. Starker Meteorismus und Spannung des Abdomens. Geringe trockene Bronchitis. Im übrigen normaler Körperbefund, normale Temperatur. Ein schleimiger Stuhl. Das Kind erhält $5<120$ konservierte Frauenmilch.

7. 1. 1910. Körpergewicht $3410 \mathrm{~g}$. Seit 2 Tagen starke Gèwichtsabnahme (270 g) und hohe T'emperatursteigerung infolge einer. Angina lacunaris. Schleimige Stühle. Das Kind trinkt schlecht. Kein Meteorismus des Abdomens mehr. Nahrung: $6 \times 100 \mathrm{Kuhmileh} 1: 1 \mathrm{mit}$ Milchzucker.

8. I. 1910. Körpergewicht $3450 \mathrm{~g}$. Von gestern auf heute Zunahme um $40 \mathrm{~g}$. Das Kind trinkt gut. Der Stuhl gebunden. Rachenbefund wieder normal. Das Allgemeinbefinden wesentlich gebessert.

10. I. 1910. Körpergewicht 3530 g. Anhaltende, gute Gewichtszunahme. Homogene Stühle, reger Appetit. Der interne Befund normal. In $10 \mathrm{~cm} \mathrm{Harn}$ $27 \mathrm{mg} \mathrm{P}_{2} \mathrm{O}_{5}$.

11. I. 1910. Körpergewicht $3530 \mathrm{~g}$. Von gestern auf heute Gewichtsstillstand bei sonst negativem Körperbefund und gutem Wohlbefinden. Stühle normal. Nahrung: $6 \times 100 \mathrm{Kuhmilch} 1: 1$ wie bisher, aber mit Saccharin.

12. I. 1910. Körpergewicht $3550 \mathrm{~g}$. Schleimige Stühle. Im $\mathrm{Harn} 37 \mathrm{mg} \mathrm{P}_{2} \mathrm{O}_{5}$.

14. I. 1910. Körpergewicht $3600 \mathrm{~g}$. Von gestern auf heute Gewichtsstillstand. Guter Appetit, homogene Stühle. Das Kind erhält $6 \times 100$ Kuhmiloh $1: 1$ mit. $3 \%$ Soxhlets Nährzucker.

17. I. 1910. Körpergewieht $3700 \mathrm{~g}$ (durchsehnittlicher Gewichtsansatz $33,3 \mathrm{~g}$ täglich). Völlig normaler Körperbefund. In $10 \mathrm{ccm} \mathrm{Harn} \mathrm{16,5} \mathrm{mg} \mathrm{P}_{2} \mathrm{O}_{5}$.

19. I. 1910. Körpergewicht $3790 \mathrm{~g}$. Völlig homogene Stühle. Das Kind ist frisch. Die Trinkmenge wird erhöht: $6 \times 120$ Kuhmilch $1: 1$ mit $3 \%$ Soxhlets Ňährzucker.

23. I. 1910. Körpergewicht $3920 \mathrm{~g}$. Andauernder, fast geradliniger, guter Gewichtsanstieg (durchschnittlich $32,5 \mathrm{~g}$ täglich). Völlig homogene Stühle. Guter Appetit. Bestes Wohlbefinden. 
Resumee: Ein in gutem Gewichtsanstieg befindlicher Säugling hat bei Halbmilch mit $3 \%$ Nährzucker eine hohe Phosphatzahl, diese hohe Zahl wird bei einer vorübergehenden Verdaungsstörung trotz Aussetzens des Kohlehydrates (Nährzucker) noch höher und sinkt bei normaler Verdaung trotz Nähruckerdarreichung wieder a uf einen kleineren Wert herab. (Fig. l.)

Keller $\mathbf{r}^{\mathbf{1}}$ ) teilte in seiner ausführlichen Studie mit, daß die künstlich genährten Säuglinge viel mehr anorganischen Phosphor im Harne ausscheiden als Brustkinder, und zwar übersteigt die Mehrausfuhr bedeutend die Mehr- 3900 einfuhr (durch die phosphorreichere Kuhmilch). Diese Béobachtung konnten auch wir bei unseren Untersuchungen bestätigen. Weiter fanden wir, daß die Phosphatausfuhr mit der steigenden Konzentration der Nahrung unverhältnismäßig stark zunimmt, sei es, daß man eine stärkere Milchkonzentration wählt, sei es, daß man Zucker oder Mehle der Nahrung zusetzt. Insbesondere soheint es, daß der Kohlehydratzufuhr eine starke anorganische Phosphorausfuhr durch den Harn entspräche, welche Steigerung in dem geringen Phosphatgehalte dieser Nährmittel nicht allein seinen Grund haben kann.

Beispiele hierfür mögen folgende Fälle bilden:

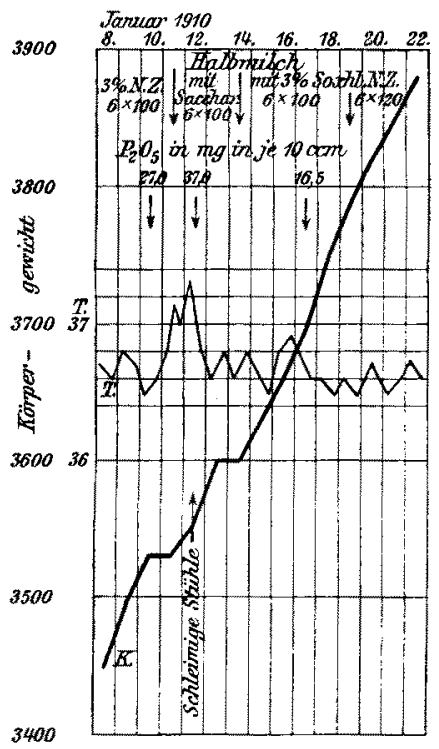

Fig. 1 .

* Fall 3. Elisabeth Buchinger, 2 Monate alt. 6 Wochen Brust, dann 2 Tage $1 / 3$ Kuhmilch. Daraufhin grüne, topfige stinkende Stühle. Auf Anraten des Arztes Kufeke mit Kuhmilch $1: 2$. Als der Stuhl nicht besser wurde, Kufekewasser. Nun besserten sich die Stühle, doch nimmt das Kind nicht zu.

31. XII. 1909. Spitalsaufnahme, 24 stündige Teediät. $1 \times 0,03$ Calomel.

1. I. 1910. Körpergewicht 3380 g. Temperatur normal. Ausgetragenes Kind, abgemagert. Am Halse und in der Leistengegend beiderseits erbsengroße Drüsen. Das Abdomen gut gespannt, leicht schmerzhaft. Kein Meteorismus. Schleimigtopfige Stühle. Sonst negativer interner Befund. Ernährung: $5 \times 100 \mathrm{Kuhmilch} 1: 2$.

4. I. 1910. Körpergewicht 3450 g. Stetige Gewichtszunahme (durchschnitt-

1) Keller, Phosphorstoffwechsel im Säuglingsalter. Zeitschr. f. klin. Med. 36, 49. 1899; derselbe, Organische Phosphorverbindungen im Säuglingsham, ihr Ursprung und ihre Bedeutung für den Stoffwechsel. Zeitschr. f. physiol. Chemie 29, 146. 1900; derselbe, Phosphor und Stickstoff im Säuglingsorganismus. Archiv f. Kinderheilk, 29, 1. 1900 . 
lich $23,3 \mathrm{~g}$ täglich). Gelbe, leicht topfige, gebundene Stühle. Guter Appetit. Normaler Körperbefund. Emährung: $5 \times 120 \mathrm{Kuhmilch} 1: 2$. In $10 \mathrm{ccm} \mathrm{Harn}$ $4 \mathrm{mg} \mathrm{P}_{2} \mathrm{O}_{\overline{5}}$.

6. I. 1910. Körpe rgewicht $3500 \mathrm{~g}$. Von gestern auf heute Gewichtsstillstand bei sonst bestem Wohlbefinden. Homogene Stïhle. Das Kind trinkt gut. Es erbält weiter wie bisher $5 \times 120$ Kuhmilch $1: 2$, von heute ab mit $3 \%$ Soxhlets Nährzucker.

12. I. 1910. Körpergewicht $3740 \mathrm{~g}$. Seit dem Nährzuckerzusatze steigt die Gewichtskurve beinahe in einer geraden Linie steil an. (Durchschnittlicher täglicher

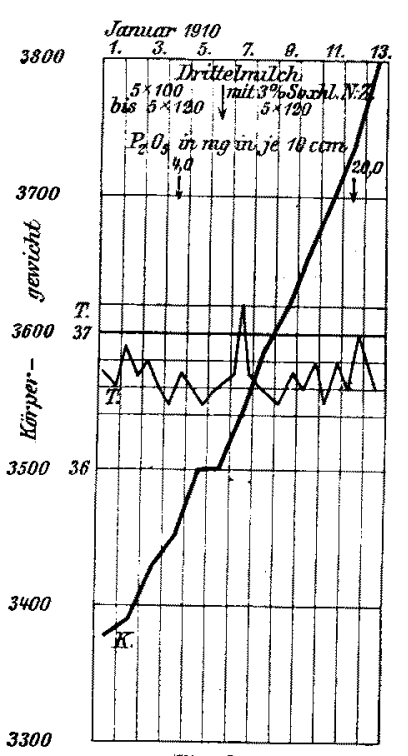

Fig. 2 . Gewichtsansatz 40 g.) Das Kind ist friseh, trinkt gut. Die Stühle gebunden, zeitweise leicht topfig, schleimig. Der übrige Körperbefund völlig normal. In $10 \mathrm{~cm} \mathrm{Harn} 20 \mathrm{mg} \mathrm{P}_{2} \mathrm{O}_{5}$.

13. I. 1910. Körpergewicht $3800 \mathrm{~g}$ (seit gestem $60 \mathrm{~g}$ Ansatz). Das Wohlbefinden anhaltend. Das Kind wird geheilt entlassen.

Resumee: Ein künstlich genährtes Kind befindet sich in guter Gewichts. zunahme, anfänglich bei reiner Drittel. kuhmileh, später beiDrittelkuhmilch mit $3 \%$ Nährzuckerzusatz. In der gesunden Milchperiode hatte es in $10 \mathrm{com} \mathrm{Harn} 4 \mathrm{mg}$ $\mathrm{P}_{2} \mathrm{O}_{5}$, inder gesunden $\mathrm{Nährzuckerperiode}$ $20 \mathrm{mg} \mathrm{P}_{2} \mathrm{O}_{5}$ in $10 \mathrm{ccm}$ Harn. Die Gewichtskurve (Fig. 2) hiorzu folgt nebenstehend.

* Fall 4. Ludwig Buchner, $3^{1 / 2}$ Monate alt, normal ausgetragenes Zwillingskind. Erhält seit 5 Wochen Kuhmilchverdünnungen; schlechte Stühle, Erbrechen.

21. XI. 1909. Körpergewicht $2860 \mathrm{~g}$, entsprechend großes, abgemagertes Kind. 2 flüssige Stühle. Die Bauchdecken leicht gespannt, kein Meteorismus. Sonst normaler Körperbefund. Seit gestern Teediä.t. Von heute ab erhält das Kind Tee mit $3 \%$ Soxhlets Nährzucker.

22. XI. 1909. Körpergewicht $2890 \mathrm{~g}$. 3 Stühle, noch immer flüssig, 2 maliges Erbrechen. Das Kind erhält 2 Magenspülungen.

23. XI. 1909. Körpergewicht $2900 \mathrm{~g}$. Der Stuhl heute homogen. Einmal noch Erbrechen. Das Kind erhält heute $7 \times 80 \mathrm{Kuhmilch} 1: 1$.

30. XI. 1909. Körpergewicht $3060 \mathrm{~g}$. Bisher ständige gute Gewichtszunahme, seit gestern eine geringe Abnahme um $10 \mathrm{~g}$. Die Stühle immer normal. Kein Erbrechen. Auch sonst das Allgemeinbefinden gut. $6 \times 100 \mathrm{Kuhmilch} 1$ : 1 .

2. XII. 1909. Körpergewicht $3140 \mathrm{~g}$. Tägliche durchschnittliche Gewichtszunahme bisher 25,5 g. Das Kind ist bei Appetit. Der intrene Befund völlig normal. In $10 \mathrm{ccm}$ Harn $5 \mathrm{mg} \mathrm{P}_{2} \mathrm{O}_{5}$.

4. XII. 1909. Körpergewicht $3160 \mathrm{~g}$. Seit gestern Gewichtsabnahme um $10 \mathrm{~g}$ bei sonst gutem Wohlbefinden. Trinkt immer aus. Stühle normal. Das Kind erhält $6 \times 100$ Kuhmilch $1: 1$ mit $3_{0}^{\circ}$ Soxhlets Nährzucker. 
10. XIX. 1909. Körpergewicht $3360 \mathrm{~g}$. Bisher dauernd gute Gewichtszunahme $\{30-50 \mathrm{~g}$ täglich). Seit gestem Gewichtsstillstand. Der interne Befund völlig normal. Das Kind ist frisch, trinkt gut. $6 \times 120 \mathrm{Kuhmiloh} 1: 1 \mathrm{mit} 3 \%$ Soxhlets Nährzucker.

12. XII. 1909. Körpergewicht $3400 \mathrm{~g}$. Neuerliche Gewichtszunahme. Das Kind trinkt immer aus. Die Stühle normal. Normaler Körperbefund. In $10 \mathrm{ccm}$ Harn $12,5 \mathrm{~m} g \mathrm{P}_{2} \mathrm{O}_{5}$.

20. XII. 1909. Körpergewicht $3680 \mathrm{~g}$. Bisher tägliche Gewichtszunahme um $20-.0 \mathrm{~g}$, seit gestern Gevichtsstillstand. Das Kind trinkt gut. Die Stühle normal, hostes: Wohlbefinden. Das Kind erhält $6 \times 120$ Kuhmileh $1: 1$ mit 3\% Kufekemehl.

22. XII. 1909. Körpergewicht $3730 \mathrm{~g}$. Gewichtzzunahne bei normalem Körperlyefund. In $10 \mathrm{cem}$ Harn $18,5 \mathrm{mg} \mathrm{P}_{2} \mathrm{O}_{5}$.

25. XII. 1909. Körpergewicht $3770 \mathrm{~g}$. Weitere Gewichtszunahme. Das Kind befindet sich wohl. Es erhält $6 \times 130 \mathrm{Kuhmilch} 1: 1$ mit 3\% Kufeke.

27. XII. 1909, Körpergewicht 3790 g. Nach einer geringen Gewichtsabnahme ron $10 \mathrm{~g}$ gestern heute neverliche Gewichtszinahme um $30 \mathrm{~g}$. Das Kind trinkt gut. Die Stühle normal. Ton heute ab $6 \times 140 \mathrm{Kuhmilch} 1: 1 \mathrm{mit}$ $3 \%$ Kufeke.

28. XII. 1909. Körpergewicht $3830 \mathrm{~g}$. Normaler Körperbefund. In $10 \mathrm{ccm}$ Harn 26,25 $\mathrm{mg} \mathrm{P}_{2} \mathrm{O}_{5}$.

2. I. 1910 . Körpergewich $4040 \mathrm{~g}$. Weiter anhaltende gute Gewichtszunahme. Das Kind ist frisch. trinkt yut. Die Stühle homogen, $1-2$ in 24 Stunden. Der interne Befund sonst notmal.

Pesnmee: Fin künstlich genährter säugling gedeiht gut bei halbrerdinnter Kuhmilch. Er erhalt nacheinander Halbmilen mit $30^{\circ}$ Soxhlets Nahrzucker and zuletzt Halbmilch mit Kufekemehl $\left(3^{\circ}, 0\right)$. Die thosphate sind wabrend der gesunden Halbmilchperiode am geringsten $(5 \mathrm{mg}$ in $10(\mathrm{~cm})$, während der gesunden Nährzuckerperiode steigen sie an $(12,5$ in $10 \mathrm{cem})$ und sind während der gesunden Mehlperiode am höchsten (anfangs 18,5, später $26,25 \mathrm{mg}$ in je $10 \mathrm{cem} H$ arn. Der Übersicht halber folgt die Kurve [Fig. 3].

Einen guten Vergleich der Phosphatausfuhr während Frauenmilohernährung, Kuhmilchernährung und Kohlehrdratdarreichung erlaubt der folgende Fall:

*Fall 5. Franz Parlik, 3 Monate alt. Erhielt 6 Wochen Brust, dann Säuslingsmileh won einer Ililchrerteilungsanstalt bis zum Tage der Aufnahme. Seit 14 Tagen trinkt das Kind sehr wenig, magert $a b$. Seit 4 Tagen Erbrechen nach jeder Nahlzeit. Zahlreiche mehr gelb verfärbte; bröckelig schleimige Stühle.

4. XI. 1909. Körpergerricht $3350 \mathrm{~g}$. Ein seinem Alter entsprechend großes Kind, ausgetragen, abgemagert. Die Haut zu bleibenden Falten abhebbar. Die Fontanelle eingesunken. Das Abdomen mäßig aufgetrieben. Durch die dünnen Bauchdecken peristaltische Bewegungen der Darmschlingen sichtbar. Das Kind erhält $5 \times 100$ Kuhmilch $(1: 2)$ mit Milchzucker, später, als das Körpergewicht nicht zunchnen will, mit Nährzucker $(3 \%)$. 


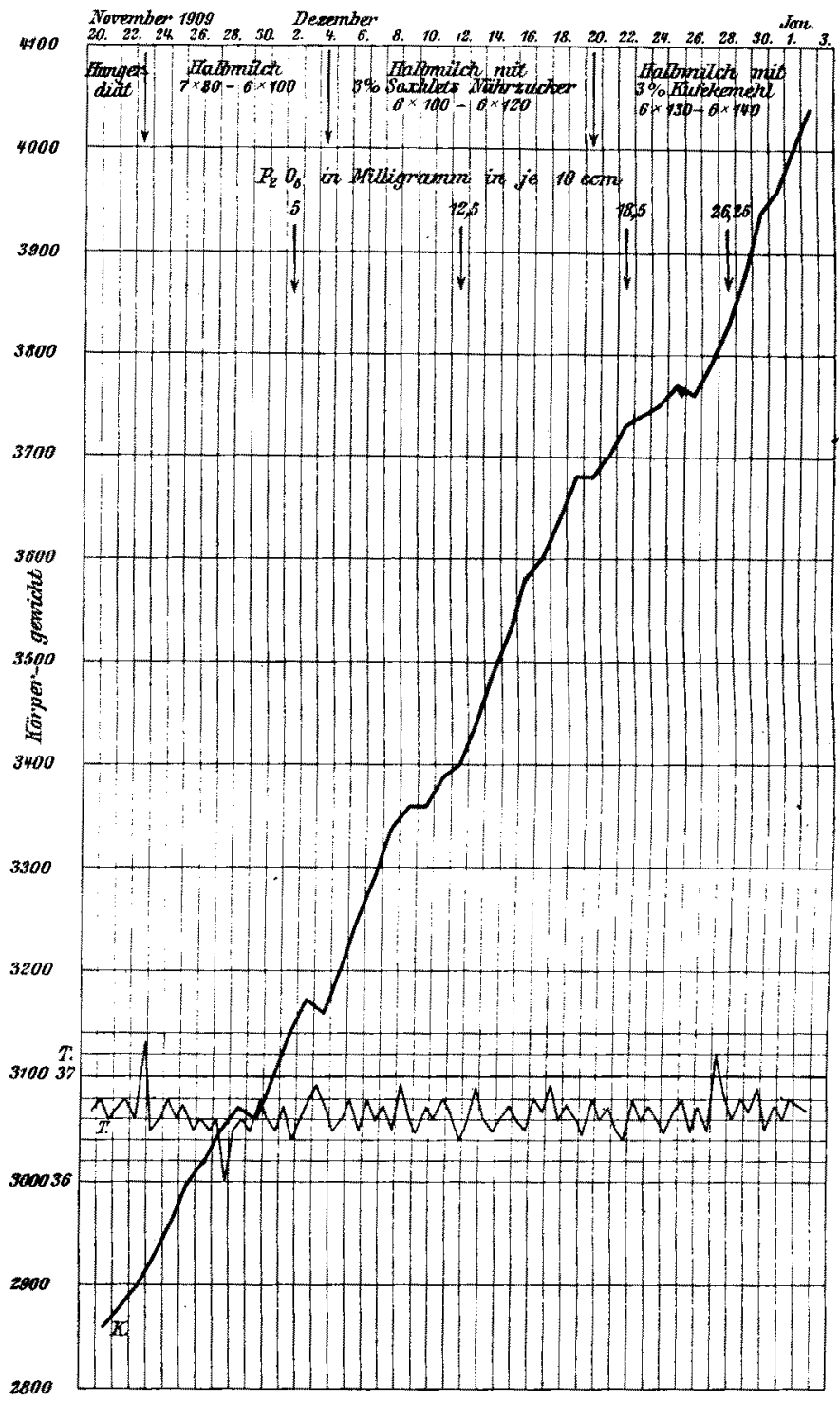

Fig. 3.

29. XI. 1909. Körpergewicht 3360 g. Die Krankheitserscheinungen sind während dieser Zeit völlig zurückgegangen. Der Appetit gut. Die Stühle homogen, nur etwas schleimig. Doch bleibt das Körpergewicht bei einigen Schwankungen ziemlich konstant. Tee; $5 \times 120$ konservierte Fravenmilch. (Bis zu 48 Tagen konserviert.) 
des anorganischen Phosphors im Harne unnatürlich ernährter Säuglinge.

2. XII. 1909. Körpergewicht $3400 \mathrm{~g}$. Langsamer Gewichtsanstieg. Appetit gut. Die Stühle etwas topfig. Der interne Befund normal. In $10 \mathrm{ccm} \mathrm{Harn}$ keine Phos phate.

9. XII. 1909. Körpergewicht $3550 \mathrm{~g}$. Anhaltende Gewichtszunahme (durchschnittlich 21,4 g täglich). Die Stühle gewöhnlich etwas toplig und schleimig. Sonst ist das Kind frisch, trinkt gut. Temperatur stets normal. In $10 \mathrm{ccm}$ Harn $1 \mathrm{mg} \mathrm{P}_{2} \mathrm{O}_{5}$.

10. XII. 1909. Körpergewicht $3580 \mathrm{~g}$. Homogene Stüle. Nahrung: $6 \times 120$ konservierte Frauenmilch.

11. XII. 1909. Körpergewicht $3650 \mathrm{~g}$. Das Kind trinkt gut. Homogene Stühle. Normaler Körperbefund. Nahrung: $4 \times 120$ konservierte Frauenmilch und $2 \times 120$ Kuhmilch 1: 1 .

12. XII. 1909. Körpergewicht 3690 g. Etwas topfige Stühle. Nahrung: $3 \times 120$ konservierte Frauenmilch und $3 \times 120 \mathrm{Kuh}$. milch $1: 1$.

13. XII, 1909. Körpergewicht $3720 \mathrm{~g}$. Normale Temperatur. Leicht topfige Stühle. Guter Appetit. Bestes Wohlbefinden. In $10 \mathrm{ccm} \mathrm{Harn} \mathrm{2,5} \mathrm{mg} \mathrm{P}_{2} \mathrm{O}_{5}$.

17. XII. 1909. Körpergewicht $3840 \mathrm{~g}$. Weiter anhaltender Gewichtsanstieg, bei homogenen stühlen. Durchschnittl. Gewichtsansatz $30 \mathrm{~g}$ täglich.

18. XII. 1909, Körpergewicht $3840 \mathrm{~g}$. Ton gestern auf heute Ge-

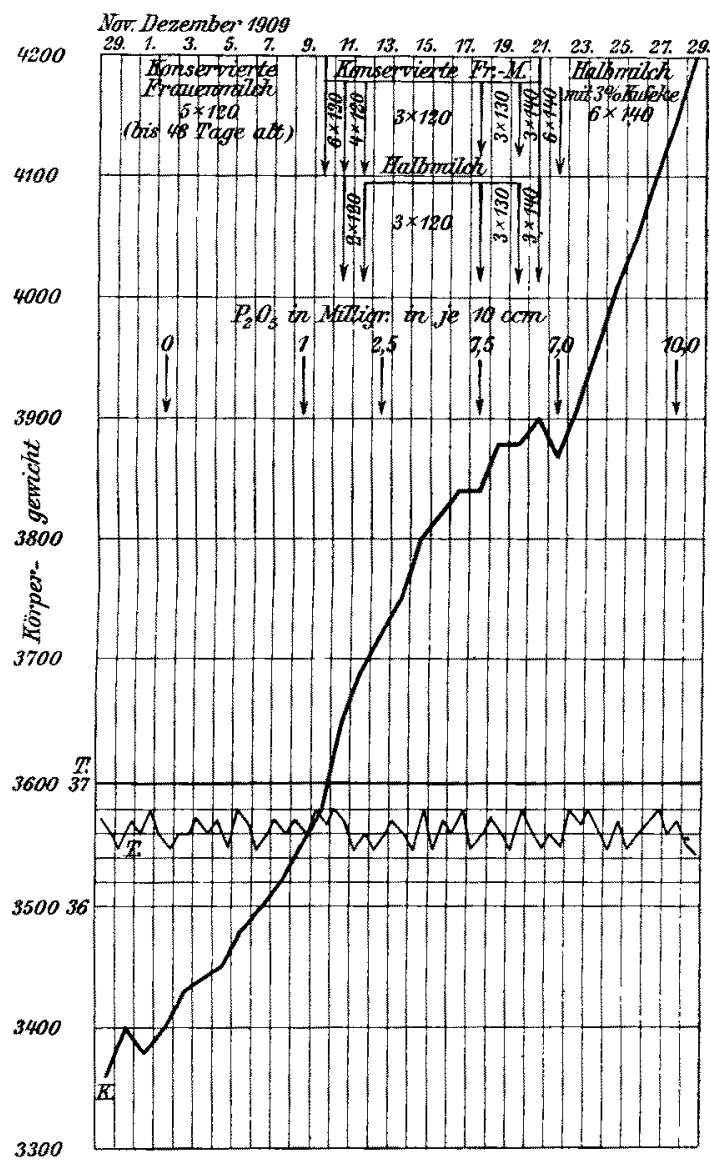

Fig. 4. wichtsstillstand. Normale Temperatur. Homogene Stühle. Das Kind ist frisch, trinkt gut. Der interne Befund völlig normal. Nahrung: $3 \times 130$ Kuhmilch $1: 1$ und $3 \times 130$ konservierte Frauenmilch. In $10 \mathrm{ccm} \mathrm{Harn}$ $7,5 \mathrm{mg} \mathrm{P}_{2} \mathrm{O}_{5}$.

20. XII. 1909. Körpergewicht $3880 \mathrm{~g}$. Von gestern auf heute wieder eimmal Gewichtsstillstand bei sonst bestem Wohlbefinden. Guter Appetit, homogene Stühle. Erhöhung der Quantität: $3 \times 140 \mathrm{Kuhmilch} 1: 1$ und $3 \times 140 \mathrm{konser}$ rierte Fravenmilch. 
21. XII. 1909. Körpergewicht $3900 \mathrm{~g}$. Das Kind erhält ron heute ab nur Kuhmilch allein, und zwar $6 \times 140(1: 1)$.

22. XII. 1909. Körpergewicht $3870 \mathrm{~g}$. Von gestern auf heute Gewichtsabnahme um $30 \mathrm{~g}$. Ein homogener, etwas schleimiger Stuhl. Appetit gut. Das Kind ist munter. Normaler Körperbefund. Temperatur normal. Therapie: $6 \times 140 \mathrm{Kuhmilch} 1: 1$ mit $3_{0}^{\circ}$ Kufeke. In $10 \mathrm{com} \mathrm{Harn} 7 \mathrm{mg} \mathrm{P}_{2} \mathrm{O}_{5}$.

28. XII. 1909. Körpergewicht $4150 \mathrm{~g}$. Anhaltender, steiler Gewichtsanstieg in fast gerader Linie (durchschnittlicher Ansatz 46,7 g täglich). Völlig homogene Stühle. Bestes Wohlbefinden. In $10 \mathrm{ccm} \mathrm{Harn} 10 \mathrm{mg} P_{2} \mathrm{O}_{5}$.

29. XII. 1909. Körpergewicht $4200 \mathrm{~g}$. Normale Temperatur. Homogener Stuhl. Appetit gut. Körperbefund normal. Das Kind wird geheilt entlassen.

Resumee: Ein bei konservierter Frauenmilch von einer Magendarmstörung genesendes Kind hat keine oder nur minimale anorganische Phosphatmengen im Harn. Beim langsamen Abstillen auf Halbmilch steigen bei gutem Gedeihen die Phosphatmengen. In einer $n u n$ folgenden $\mathrm{K}$ ufekeperiode mit $\mathrm{Halb}_{\mathrm{b}} \mathrm{ilch}$ steigen die $\mathrm{Phos}$ phatmengen noch mehr an. Eine bessere Übersicht über diese Verhältnisse gewährt die Kurve (Fig. 4).

Bei hohen und mittleren Phosphatzahlen fanden wir zuweilen ein unmotiviertes Schwanken. Ein Beispiel hierfür bildet folgender Fall:

*Fall 6. Felix Osterberger, 5 Monate alt. Das Kind hat in letzter Zeit Zweidrittelkuhmilch erhalten. Seit \& Tagen Ficber, Wnruhe, starker Husten. Dic Stühle topfig, gewwhnlich 3 täglich.

9. I. 1910. Aufnahme. 24stündige Teediät.

10. I. 1910. Körpergewicht $4750 \mathrm{~g}$. Entsprechend grobes, ausgetragenes Kind, ron mäßig gutem Ernährungszustand. Rachitisch veränderter Knochenbau. Vergröberte Drüsen beiderseits am Halse, in der Axilla und besonders in inguine. Metcorismus und Spannung des Abdomens. Linksseitige feuchte Bronchitis. Coryza. Temperatur normal. Das Kind erhält $4 \div 120 \mathrm{Kubmilch} 1: 1$.

13. I. 1910. Körpergewicht $4640 \mathrm{~g}$. Das Kind nimmt ständig ab. Die Bronchitis fast völlig gebessert. Coryza geschwunden. Kein Meteorismus des Abdomens mehr. Appetit gut. Die Stühle homogen. Nahrung: $\cdot 5 \times 120 \mathrm{Kuhmilch} 1: 1 \mathrm{mit}$ $3 \%$ Phosphatine-Falières.

14. I. 1910. Körpergewicht $4530 \mathrm{~g}$ (Abnahme $110 \mathrm{~g}$ ). Homogene Stühle, Das Kind trinkt gut. Normaler Körperbefund. Erhöhung der Trinkmenge: $5 \times 150$ Kuhmilch 1 : 1 mit 3\%o Phosphatine. In $10 \mathrm{com} \mathrm{Harn} 13 \mathrm{mg} \mathrm{P}_{2} \mathrm{O}_{5}$.

17. I. 1910. Körpergewicht $4630 \mathrm{~g}$. Andauernder, guter Gewichtsanstieg (durchschnittlich $33,3 \mathrm{~g}$ täglich). Völlig homogene Stühle. Bestes Wohlbefinden. In $10 \mathrm{~cm}$ Harn $57,5 \mathrm{mg} \mathrm{P}_{2} \mathrm{O}_{5}$.

19. I. 1910. Körpergewicht $4650 \mathrm{~g}$. Von gestern auf heute Abnahme um $10 \mathrm{~g}$. Reger Appetit. Völlig normaler Körperbefund. Die Stühle homogen. Vermehrung der Mahlzeiten auf: $6 \% 150$ Kuhmileh $1: 1$ mit $3 \%$ Phosphatine.

24. I. 1910. Körpergewicht $4850 \mathrm{~g}$. Stetiger Gewichtsanstieg, in steiler, fast geradliniger Kurve. Das Kind ist munter, hat guten Appetit. Die Stühle homogen. Der interne Befund normal. In $10 \mathrm{ecm}$ Harn $32 \mathrm{mg} \mathbf{P}_{2} \mathrm{O}_{5}$.

25. I. 1910. Körpergewicht $4890 \mathrm{~g}$. Das Wohlbefinden hält an. 
Resumee: Fin bei Halbmilch mit 30: Phosphatinemehl (Falicres) in gutem Gewichtsanstieg befindlicher Säugling hat hohe Phosphat. zahlen. Bei gleichbleibendem Ernährungszustand schwanken die Phosphatzahlen zwischen mittleren und hohen Werten.

Untenstehend die Gewichtskurve (Fig. 5).

Die im Vorausgeschickten erwähnte Schwankung in den Zahlen kann nicht allein dadurch erklärt werden, daB wir nicht die 24 stündige Harnportion untersuchten, da unsere daraufhin gerichteten Untersuchungen zeigten, daß man für diese rein klinischen, nur auf grobe Veränderungen bezughabenden Harnproben mit Berechtigung eine beliebige größere Harmportion wählen kann. Dies ist ja das Wertvolle und Wesentliche an jenen Proben, daß man sich rasch aus der Harnuntersuchung einen die Klinik unterstützenden Befund verschaffen kann, sonst hinkt der Harnbefund dem Ablaufe der Ereignisse am Krankenbett nach.

Nachfolgender Fall zeigt, wie gut und für klinische Zwecke hinreichend genau die ein-

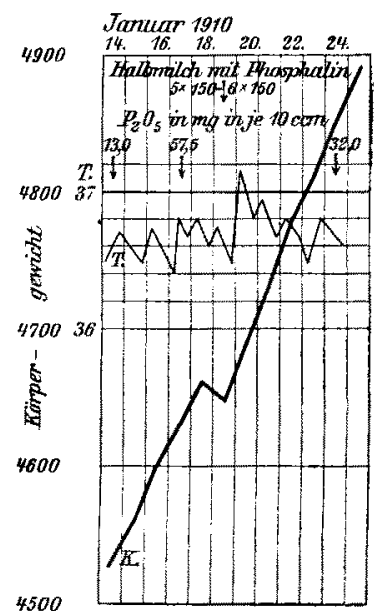

Fig. 5. zelnen Zahlen aus beliebigen kleineren oder größeren Harnportionen mit den Zahlen aus der 24 stündigen Menge übereinstimmen.

*Fall 7. Josef V., $3^{1 / 2}$ Monate alt, während des Versuches magendarmgesund. Das Kind erhält vom 12. VIII.-20. VIII. $6 \times 110$ Liebigsuppe 1:2. Während der ganzen Beobachtungszeit nahm das Kind bei homogenen Stühlen täglich regelmäßig um durchsehnittlich $16 \mathrm{~g}$ zu. Die $\mathrm{P}_{2} \mathrm{O}_{5}$-Werte in je $10 \mathrm{ccm}$ Harn beliefen sich in der Periode der Drittel-Liebigsuppe in verschiedenen aufeinanderfolgenden Zeiten im:

12 stundigen Naehthara in der teit vom 11. FIII, bis 12. VIIL, von 6 thr abends bis 6 Uhr morgens

\section{5,9 Milligramm $\mathrm{P}_{2} \mathrm{O}_{5}$} Vormittagshamn
am 12. VIII.

4,7 Milligramm $\mathrm{P}_{2} \mathrm{O}_{5}$
Abendham am 12. VIII.

5,5 Milligr. $\mathrm{P}_{2} \mathrm{O}_{5}$

Mittagsharn am 13. VIII. In der 24 stündigen Menge: $\left(=215 \mathrm{~cm}^{3}\right.$ vom 17 . vill, bis 18 . VIII. 4,0 Milligr. $\mathrm{P}_{2} \mathrm{O}_{3}$ 4,4 Muligramm $\mathrm{P}_{2} \mathrm{O}_{5}$

Wir bemerken also während einerganzen Woche einefür unsere klini. schen Zwecke genügend genaue Ċbereinstimmung $z$ wischen den $Z$ ahlen verschiedener Portionen und der 12 - sowie 24 stündigen Harn. menge.

Dasselbe Kind erhielt bei unverändertem Wohlbefinden rom 20.-26. VIII. eine konzentriertere Nahrung in Form von $6 \times 100$ Liebigsuppe $2: 3$. Die $\mathrm{P}_{2} \mathrm{O}_{\tilde{a}}$ - 
34 G. Kaminer und E. Mayerhofer: Über den klinischen Wert der Bestimmung

Mengen stiegen während dieser Zeit der Darreichung von $2 / 5$ Liebigsuppe auf beinahe das Doppelte an. Die Werte betrugen in je $10 \mathrm{~cm}$ Harn beliebiger Harn. portionen am:

20. VIII.

22. VIII.

24. VIII.

7,75 Milligr. $\mathrm{P}_{2} \mathrm{O}_{5}$

9,75 Milligr. $\mathrm{P}_{2} \mathrm{O}_{5}$

7,5 Milligr. $\mathrm{P}_{2} \mathrm{O}_{5}$

Auch aus diesen Zahlen erkennen wir, daB das Ansteigen der Phosphatzahlen bei Erhöhung der Nahrungskonzentration genügend genau an den einzelnen Harnportionen kenntlich wird.

Im Anhange des ersten (klinischen) Teiles dieser Arbeit sind noch weitere 31 Fälle mitgeteilt, deren tabellarische Aufzählung genügt.

Das Resumee über diese praktisch-klinischen Untersuchungen ist folgendes:

A. Die anorganischen Phosphate steigen im Harne bei:

1. Verdauungsstörungen (Fall 1, 2, 33).

2. Parenteraler Störung (Fall 31).

3. Erhöhung der Konzentration der künstlichen Nahrung:

a) beim Ưbergang von der Teediät zu irgendeiner künstlichen $\mathrm{Nah}$ rung (Fall 26),

b) bei Verstärkung der Kuhmilchkonzentration (Fall 26, 35, 38).

c) bei Zuckerzusatz (Fall 3, 4, 14, 22, 24, 26, '28),

d) bei Mehlzusatz (Fall 4, 5, 16, 18, 33, 35).

4. Beim Übergang von Frauenmilch zu einer Kuhmilch (Fall 5, 36) oder Kuhmilchmehlmischung (Fall 30, 3I).

B. Die anorganischen Phosphate sinken im Harn bei:

1. Heilung einer Magendarmstörung (Fall 21, 37),

2. Heilung einer parenteralen Störung (Fall 31),

3. Verminderung der Konzentration der Nahrung (Fall 9, 23).

4. Erreichung des Hungerzustandes (Fall.27).

C. Diese angeführten RegelmäBigkeiten werden bei der überwiegenden Anzahl von Fällen beobachtet.

Selten findet man ein unmotiviertes Schwanken in den Phosphatzahlen einzelner Harnportionen (Fall 6, 15). In jenen Ausnahmefällen müBte man größere Harnportionen, am besten die 24 stündigen Mengen untersuchen, obwohl für klinische Zwecke eine meist genügend genaue Úbereinstimmung der einzelnen Harnportionen mit der 12- und 24stündigen Harnmenge gefunden worden ist (Fall 7).

Doch bleibt noch immer eine geringe Anzahl von Fällen übrig, bei denen bei oberflächlicher Beurteilung der Titrationsresultate unerklärliche UnregelmäBigkeiten vorhanden sind. Ein großer Teil dieser UnregelmäBigkeiten wird im II. Teil dieser Arbeit erklärt. 


\begin{tabular}{|c|c|c|c|c|c|}
\hline $\begin{array}{c}\mathbf{N r} \\
*\end{array}$ & Name, Alter & Ernährungsweise & $\begin{array}{c}\text { Daten } \\
\text { ubber das Gedeihen }\end{array}$ & $\begin{array}{c}\text { Milligramm } \\
\mathrm{P}_{2} \mathrm{O}_{5} \text { in je } 10 \mathrm{ccm} \\
\text { Harn }\end{array}$ & Anmerkung \\
\hline 8 & $\begin{array}{c}\text { Marie Lagler } \\
4 \text { Monate }\end{array}$ & $\begin{array}{l}\text { Seit 16. VIT, bis } \\
\text { 20.VIII. 1910 Halbmilch } \\
\text { mit } 1 \% \text { Hafermehlzu- } \\
\text { satzy später } 2 / 3 \text { Mileh } \\
\text { mit } 1 \% \text { Hafermehl }\end{array}$ & $\begin{array}{l}\text { Bei guten Stihlen und } \\
\text { normalem klinischen } \\
\text { Verhalten in } 35 \text { Tagen } \\
\text { eine durchsehnittliche } \\
\text { Zunahme von } 25,7 \mathrm{~g}\end{array}$ & 12. VIII. 23,0 & $\begin{array}{c}\text { Hohe Zahl } \\
\text { während Halb. } \\
\text { milch mit 1\% } \\
\text { Hafermehl }\end{array}$ \\
\hline 9 & $\begin{array}{l}\text { Franziska } \\
\text { Matejicek } \\
6 \text { Wochen }\end{array}$ & $\begin{array}{l}\text { Seit 5. VIII. bis 20. VIII. } \\
\text { Liebigsuppe, anfangs } \\
1: 2 \text {, später } 1: 1\end{array}$ & $\begin{array}{l}\text { In } 15 \text { Tagen guten } \mathrm{Ge} \text { - } \\
\text { deihens tägliche } \\
\text { durchschnittliche } \mathrm{Zu} \text { - } \\
\text { nahme von } 28 \mathrm{~g}\end{array}$ & $\begin{array}{l}\text { 13. VII. } 7 \\
20 . \text { VIII. } 7,5\end{array}$ & $\begin{array}{l}\text { Mittlere Zahl } \\
\text { bei Liebigsuppe } \\
1: 2 \text { und } 1: 1\end{array}$ \\
\hline & & $\begin{array}{l}\text { Seit 20.VII. Halbmilch } \\
\text { mit } 1 \% \text { Hafermeblzu- } \\
\text { satz bis } 5 . \mathrm{IX} \text {. }\end{array}$ & $\begin{array}{c}\text { In } 16 \text { Tagen } 11,8 \mathrm{~g} \\
\text { durchschnittliche } \\
\text { Tageszunabme; nor- } \\
\text { males klinisches Ver- } \\
\text { halten }\end{array}$ & 5. IX. & $\begin{array}{l}\text { Sinken der } \\
\text { Phosphate bei } \\
\text { geringerer } \\
\text { Konzentration } \\
\text { der Nahrung }\end{array}$ \\
\hline 10 & $\begin{array}{l}\text { Anna Dums } \\
5 \text { Monate }\end{array}$ & $\begin{array}{l}\text { Seit 11. VIII bis } \\
\text { 28. VIII. Liebigsuppe } \\
1: 1\end{array}$ & $\begin{array}{l}\text { In } 17 \text { Tagen regel- } \\
\text { mäBigen Gedeihens } \\
\text { tägliche durch- } \\
\text { sehnittliche Zunahme } \\
\text { von } 30,6 \mathrm{~g}\end{array}$ & $\begin{array}{l}\text { 13. VIII. } 5 \\
\text { 20. VIII. } 16,0\end{array}$ & $\begin{array}{l}\text { Steigt während } \\
\text { der Darreichung }\end{array}$ \\
\hline 11 & $\begin{array}{l}\text { Mathilde } \\
\text { Hajek } \\
5 \text { Monate }\end{array}$ & $\begin{array}{c}\text { Seit 20. } 1 \text { X. bis 6.X. } \\
19095 \times 3: 1 \text { Nestle } \\
(8 \%) 1 \times \text { Griebbre }\end{array}$ & $\begin{array}{l}\text { In } 16 \text { Tagen der Ge- } \\
\text { sundheit regelmäBige } \\
\text { tägliche durch- } \\
\text { schnittiche Zunahme } \\
\text { von } 35 \mathrm{~g}\end{array}$ & $\begin{array}{l}\text { 1. IX. } \\
\text { 2. IX. }\end{array}$ & $\begin{array}{c}\text { Hohe Zahlen bei } \\
\text { konzentrierter } \\
\text { Nahrung }\end{array}$ \\
\hline 12 & $\begin{array}{l}\text { Franz Toppler } \\
31 / 2 \text { Monate }\end{array}$ & $\begin{array}{c}\text { Seit } 31 \text {. VTI bis } 14 \text {.VIII. } \\
1: 1 \text { mit } 1 \% \text { Hafermehl- } \\
\text { zusatz }\end{array}$ & $\begin{array}{c}\text { Ein chronischer } \\
\text { Atrophiker nimmt bei } \\
\text { homogenen Stühlen in } \\
14 \text { Tagen täglich um } \\
17,1 \mathrm{~g} \mathrm{zu}\end{array}$ & $\begin{array}{ll}\text { 8. VIII. } & 7 \\
\text { 13. VIII. } & 8 \\
\text { 14. VIII. } & 2,75\end{array}$ & $\begin{array}{c}\text { Mittlere Zahl } \\
\text { bei Halbmileh } \\
\text { und Hafermehl }\end{array}$ \\
\hline 13 & $\begin{array}{l}\text { Juliane Houf } \\
6 \text { Monate }\end{array}$ & $\begin{array}{c}\text { Seit 24. VII. bis 17.VII. } \\
\text { Halbmilch mit } 3 \% \\
\text { Kufeke }\end{array}$ & $\begin{array}{c}\text { Bei homogenen } \\
\text { Stühlen durchschnitt- } \\
\text { liche tägliche Zunahme } \\
\text { um } 21,7 \mathrm{~g}\end{array}$ & 13. vur. 5,6 & $\begin{array}{c}\text { Mittlere Kahl } \\
\text { bei Halbmilch } \\
\text { und Kufekemenl }\end{array}$ \\
\hline \multirow[t]{2}{*}{14} & \multirow[t]{2}{*}{$\begin{array}{c}\text { Marie } \\
\text { Brennessel } \\
1 \text { Monat }\end{array}$} & $\begin{array}{l}\text { Vom 16. XII. bis } \\
\text { 29. XII. } 1909 \text { Drittel- } \\
\text { milch }\end{array}$ & $\begin{array}{c}\text { Bei stets dyspeptischen } \\
\text { Stülen befriedigende } \\
\text { Gewichtszunahmen, im } \\
\text { Durchschnitt } 13,8 \mathrm{~g} \\
\text { pro Tag }\end{array}$ & $\begin{array}{ll}\text { 22. XII. } & 3,0 \\
\text { 29. XII. } & 2,0\end{array}$ & $\left\{\begin{array}{c}\text { Kleine Zamlen } \\
\text { bei Drittel- } \\
\text { Kuhmilch }\end{array}\right.$ \\
\hline & & $\begin{array}{c}\text { Vom 29. XI. } \\
\text { Drittelmilch mit } 3 \% \\
\text { Nahrzuckerzusatz; } \\
\text { schlieBlich bei } 7 \times 100 \\
\text { 1:2 mit 8\% Nährzucker }\end{array}$ & $\begin{array}{l}\text { Die bisher dyspepti- } \\
\text { schen Stühle bessern } \\
\text { sich, die Gewichtskurve } \\
\text { wird viel regelmäßiger; } \\
\text { die durchschnittlichen } \\
\text { Tageszunahmen heben } \\
\text { sich in den letzten } 3 \\
\text { Wochen aut } 30,5 \mathrm{~g}\end{array}$ & $\begin{array}{l}\text { 4. I. } 1910 \quad 3,5 \\
\text { 12. I. } 30,5\end{array}$ & $\left\{\begin{array}{c}\text { Phosphate } \\
\text { steigen im } \\
\text { Verlaufe der } \\
\text { Kohlehydrat- } \\
\text { darreichming }\end{array}\right.$ \\
\hline
\end{tabular}




\begin{tabular}{|c|c|c|c|c|c|c|}
\hline Nir. & Nane, Alter & Eraährungsweise & $\begin{array}{c}\text { Daten } \\
\text { uber das Gedeihen }\end{array}$ & $\begin{array}{r}\text { Mulligran } \\
\mathrm{P}_{2} \mathrm{O}_{5} \text { in je } 1 \\
\text { Harn }\end{array}$ & $\begin{array}{l}\mathrm{mm} \\
10 \mathrm{ccm}\end{array}$ & Anmerkung \\
\hline 15 & $\begin{array}{c}\text { Rudolf } \\
\text { Havelka } \\
5 \text { Monate }\end{array}$ & $\begin{array}{c}\text { Yom 25. I. bis } 3.1 \mathrm{r} \\
19105 \times 1501: 1 \mathrm{mit} \\
3 \% \text { Nährzucker }\end{array}$ & $\begin{array}{c}\text { Bei ganz homogenen } \\
\text { Stihlen (regelmälig } 1 \times \\
\text { täglich, ein einziges Mal } \\
2 \times \text { ) geradinige Kurve } \\
\text { mitdurchschnittlichem } \\
\text { täglichen Ansatz von } \\
27,7 \mathrm{~g} \text { in den letzten } \\
9 \text { Tagen }\end{array}$ & $\begin{array}{l}27 . x .1910 \\
2 . \mathrm{Ir} .\end{array}$ & $\begin{array}{r}9,5 \\
18,0\end{array}$ & $\begin{array}{c}\text { Zahlen steigen } \\
\text { während eines } \\
\text { Halbmileh- } \\
\text { kohlehydrat- } \\
\text { futterung }\end{array}$ \\
\hline 16 & $\begin{array}{l}\text { Hermine Sejna } \\
6 \text { ISonate }\end{array}$ & $\begin{array}{c}\text { Vom 24. I. bis } 25 \text {. I. } \\
19105 \times 1001: 1\end{array}$ & $\begin{array}{l}\text { In diesen } 4 \text { Tagen bei } \\
\text { befriedigenden Stuihlen } \\
\text { ein Durchschnittsan- } \\
\text { satz von } 17,5 \mathrm{~g}\end{array}$ & 25. T. 1910 & 7,5 & $\begin{array}{l}\text { Mittlere Zahl } \\
\text { bei Halbmilch }\end{array}$ \\
\hline & & $\begin{array}{c}\text { Fom 28. I. bis 5. II. } \\
5 \times 1001: 1 \text { mit } 3 \% \\
\text { Nährucker }\end{array}$ & $\begin{array}{l}\text { Bei Besserung der } \\
\text { Stiuhle erhebt sich der } \\
\text { tägliehe Durchsohnitts- } \\
\text { ansatz auf } 22,5 \mathrm{~g} \text { in den } \\
\text { letzten } 8 \text { Tagen }\end{array}$ & $\begin{array}{l}\text { 31. I. } \\
\text { 3. II. }\end{array}$ & $\begin{array}{r}13,0 \\
5,0\end{array}$ & $\begin{array}{l}\text { Iuitiale Zache } \\
\text { nach Zusatz von } \\
\text { enem Kohle- } \\
\text { hydrat (siebt } \\
\text { Abschnitt II) }\end{array}$ \\
\hline : & & $\begin{array}{c}\text { Fon 5. II. an wird } \\
\text { Hafermehl }(1 \%) \mathrm{zu} \\
5 \times 1002: 1 \text { hinzu- } \\
\text { gegeben }\end{array}$ & $\begin{array}{l}\text { Nach anfängl. Sinken } \\
\text { der Kurve steigt sie seit } \\
\text { 6 Tagen wieder regel- } \\
\text { mäBig um den Durch. } \\
\text { schnitt von } 13,3 \mathrm{~g} \text { an }\end{array}$ & 15. II. & $15_{y} 0$ & $\begin{array}{l}\mathrm{P}_{2} \mathrm{O}_{5} \text { steigt bei } \\
\text { Erhohung der } \\
\text { Kohlehydrat- } \\
\text { konzentration }\end{array}$ \\
\hline$\vdots$ & & $\begin{array}{l}\text { Vom } 15 . \text { II. bis } 26 \text {. If. } \\
\text { erhalt das Kind } s \times 100 \\
2: 1 \text { mit } 1 \% \text { Hafermehl } \\
\text { und } 100 \mathrm{~g} \text { Griesbrei }\end{array}$ & $\begin{array}{l}\text { Die Gewichtskurve } \\
\text { nimmt einen regelmä* } \\
\text { Bigen und steileren Ver- } \\
\text { laut. In diesen } 11 \text { Tagen } \\
\text { ein täglicher Durch- } \\
\text { sehnittsansatz von } \\
\quad 31,9 \mathrm{~g}\end{array}$ & 21. II. & 25,0 & 10 \\
\hline 17 & $\begin{array}{c}\text { Marie Kasses } \\
6 \text { Monate }\end{array}$ & $\begin{array}{l}\text { Vom 1. II. bis 13. IJ. } \\
19106 \times 1002: 1 \text { mit } \\
3 \% \text { Phosphatine }\end{array}$ & $\begin{array}{l}\text { Bef hornogenen Mehl- } \\
\text { stihlen eine regelmä- } \\
\text { Bige Kurve; in diesen } \\
\text { 12Tagen täglich dureh- } \\
\text { schnittlicher Ansatz } \\
\text { von } 30 \mathrm{~g}\end{array}$ & $\begin{array}{l}\text { 3. II. } 1910 \\
9 . \mathrm{Ir}\end{array}$ & $\begin{array}{l}17 \\
15\end{array}$ & $\begin{array}{l}\text { Durehschnitts- } \\
\text { wertefür:a Milch } \\
\text { und Mebizusatz }\end{array}$ \\
\hline 18 & $\begin{array}{c}\text { Max } \\
\text { Hubsehmann } \\
10 \text { Monate }\end{array}$ & $\begin{array}{l}\text { Vom 2. III. bis } 22 . \text { III. } \\
19105 \times 1502: 1 \text { und } \\
2 \times 150 \text { GrieBbrei }\end{array}$ & $\begin{array}{l}\text { In } 20 \text { Tagen ein durch- } \\
\text { sehnithlieher Tagesar- } \\
\text { satz von } 20 \mathrm{~g} \text { bei nor- } \\
\text { malem klinisehen Ver- } \\
\text { halten }\end{array}$ & 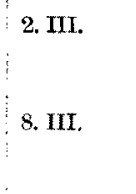 & 47,0 & $\begin{array}{l}\left\{\begin{array}{l}\text { Zahl aus der } \\
\text { Vorperiode }\end{array}\right. \\
\left\{\begin{array}{l}\text { Ansteigen der } \\
\mathrm{P}_{2} \mathrm{O}_{5}-\text { Werte bei } \\
\text { Zunahme der } \\
\text { Konzentration } \\
\text { der Nahrung }\end{array}\right.\end{array}$ \\
\hline 19 & $\begin{array}{c}\text { Franz } \\
\text { Kerschbaum } \\
6 \text { Monate }\end{array}$ & $\begin{array}{c}\text { Yom } 5 . \text { III. bis } 13 . \text { IIT. } \\
19106 \times 1802: 1 \text { mit } \\
3 \% \text { Soxhlets Nahr- } \\
\text { zucker }\end{array}$ & $\begin{array}{l}\text { Geradlinig ansteigende } \\
\text { steile Kurve mit einem } \\
\text { durchschnittlichen } \\
\text { Tagesausatz von } 55 \mathrm{~g}\end{array}$ & 6.III. 1910 & 10,0 & $\begin{array}{l}\text { Durchschnitts- } \\
\text { Wert für } \\
{ }^{2} \text { Milch und } \\
\text { Nährzucker }\end{array}$ \\
\hline 20 & $\begin{array}{c}\text { Ernst } \\
\text { Himmelreich } \\
3 \text { Mouate }\end{array}$ & $\begin{array}{l}\text { Vom } 15 . \text { III. bis } 6 . \text { IV. } \\
1910 \text { Halbmilch mit } \\
1 \% \text { Hafermehlausatz } \\
(5 \times 1330 \text { bis } 6 \times 150)\end{array}$ & $\begin{array}{c}\text { Befriedigende Kurve, } \\
\text { homogene Stühle, nur } \\
\text { 2mal erbrochen. Tag- } \\
\text { liche Durchschnittszu- } \\
\text { nahme von } 15,9 \mathrm{~g}\end{array}$ & $\begin{array}{l}\text { 16. III.1910 } \\
\text { ?8. III. }\end{array}$ & $\begin{array}{r}09,0 \\
5,0\end{array}$ & \\
\hline
\end{tabular}




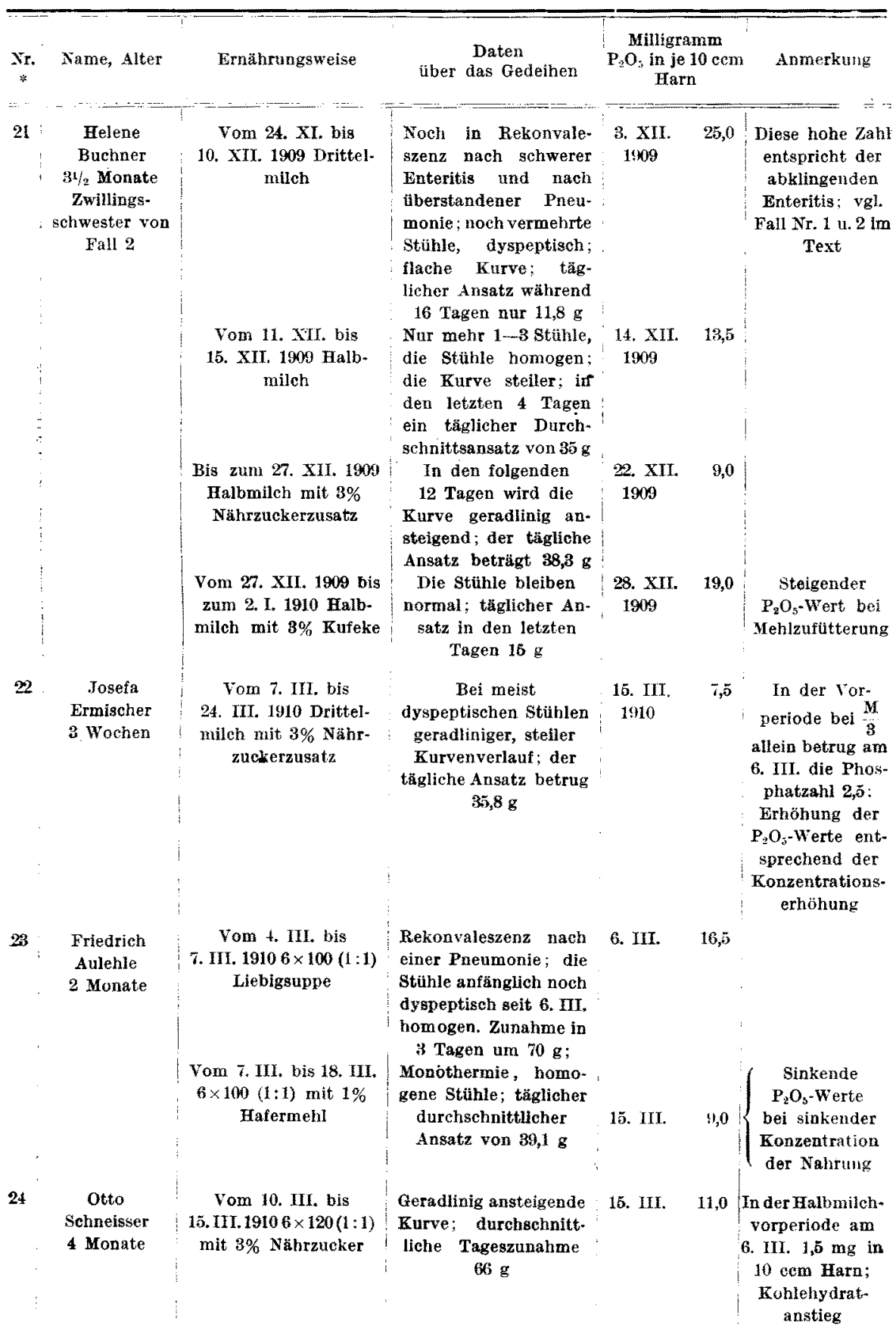




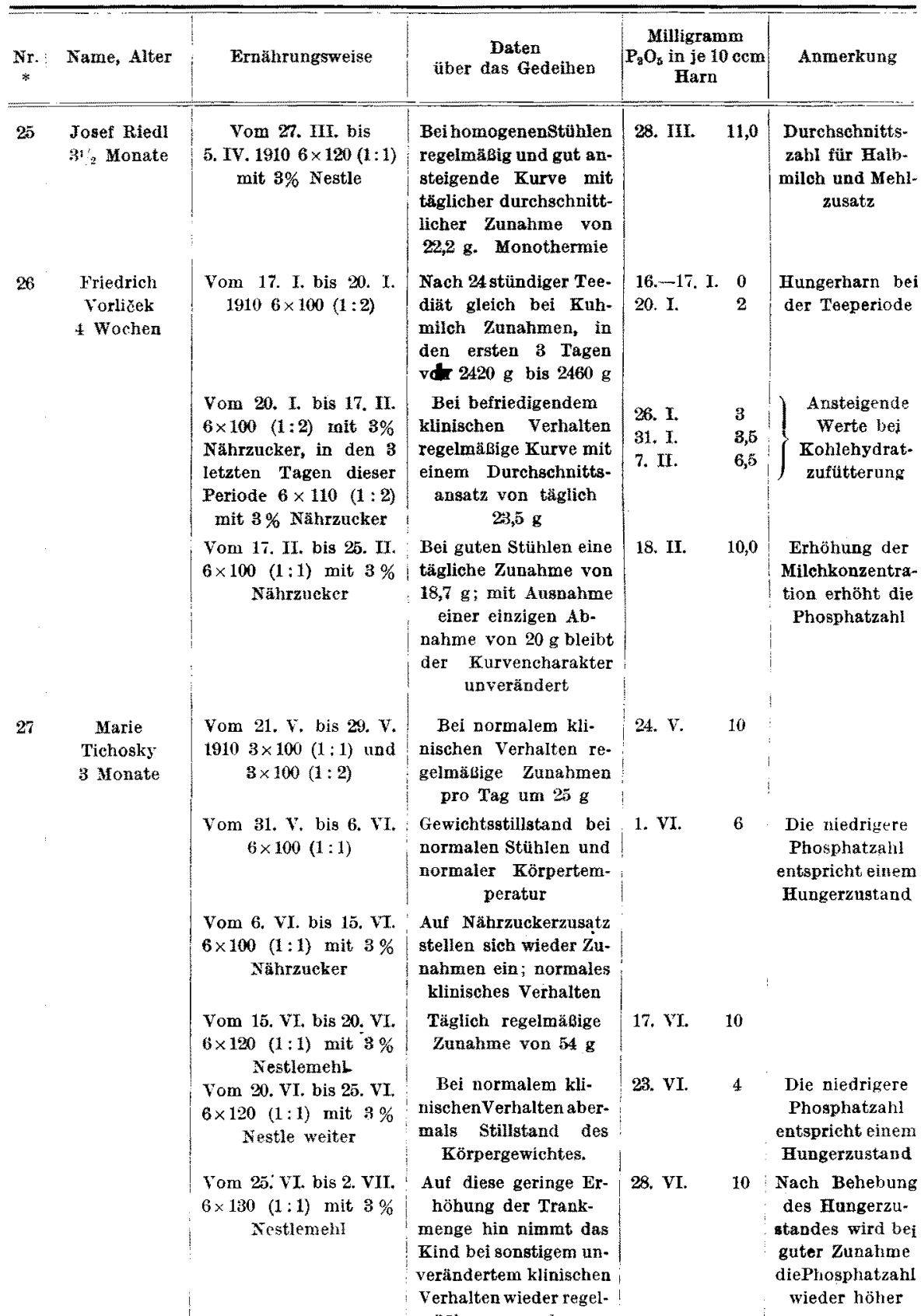


des anorganischen Phosphors im Harne unnaturlich ernährter Säuglinge.

\begin{tabular}{|c|c|c|c|c|c|c|}
\hline $\begin{array}{l}\text { Nr. } \\
\text { * }\end{array}$ & Name, Alter & Ermährungsweise & $\begin{array}{l}\text { Daten } \\
\text { über das Gedeihen }\end{array}$ & $\begin{array}{r}\text { Milligra } \\
\mathrm{P}_{2} \mathrm{O}_{5} \text { in je } \\
\mathrm{Har}\end{array}$ & $\begin{array}{l}\operatorname{man} \\
10 \mathrm{com}\end{array}$ & Anmerkung \\
\hline \multirow[t]{2}{*}{$28:$} & \multirow[t]{2}{*}{$\begin{array}{l}\text { Josef Elkers } \\
\overline{0} \text { Wochen }\end{array}$} & $\begin{array}{l}\text { Vom 27. V. } 1010 \text { bis } \\
\text { 4. VII. } 7 \times 80(1: 2)\end{array}$ & $\begin{array}{l}\text { Bel homogeneostuhlen } \\
\text { und normaler Körper- } \\
\text { temperatur täglich re- } \\
\text { gelmäßig um } 10 \mathrm{~g}\end{array}$ & 28. VI. & 3,5 & \\
\hline & & $\begin{array}{l}\text { Vom } 4 . \text { VIl. bis } 16 . \text { VII. } \\
7 \times 80(1: 2) \text { mit } 3 \% \\
\text { Năhruckerzusatz }\end{array}$ & $\begin{array}{l}\text { Bei normalem kli- } \\
\text { nischen Verhalten ge- } \\
\text { radlinig ansteigende } \\
\text { Kurve; tägliche } \mathrm{Zu}- \\
\text { nahme von } 27,6 \mathrm{~g}\end{array}$ & $\begin{array}{l}\text { 6. VII. } \\
\text { 12. VII. }\end{array}$ & $\begin{array}{l}7,5 \\
6\end{array}$ & $\begin{array}{c}\text { Ansteigende } \\
\mathrm{P}_{z} \mathrm{O}_{5} \text {-Werte bei } \\
\text { ansteigender } \\
\text { Konzentration }\end{array}$ \\
\hline 29 & $\begin{array}{l}\text { Marie Stauber } \\
3 \text { Monate }\end{array}$ & $\begin{array}{c}\text { Vom 12. XII. bis } 22 \text { XII. } \\
19095 \times 130 \text { bis } 5 \times 140 \\
(2: 1) \text { mit } 3 \% \text { Kufeke- } \\
\text { meln }\end{array}$ & $\begin{array}{c}\text { Bei homogenen Mehl- } \\
\text { strïhlen eine tägliche } \\
\text { regelmäBige Zunahme } \\
\text { von } 15,0 \mathrm{~g}\end{array}$ & $\begin{array}{l}\text { 14. XII. } \\
1909 \\
22 . \text { XII. } \\
1909\end{array}$ & $\begin{array}{l}30,0 \\
8\end{array}$ & $\begin{array}{c}\text { Initiale Kohle- } \\
\text { hydratzahl } \\
\text { (siebe Ab- } \\
\text { schnitt } \Pi \text { ) }\end{array}$ \\
\hline \multirow[t]{2}{*}{30} & \multirow[t]{2}{*}{$\begin{array}{l}\text { Nikolaus } \\
\text { Nikolaijevič } \\
6 \text { Monate, } \\
\text { Zwilling }\end{array}$} & $\begin{array}{l}\text { Bis zum } 10 . \text { WIII. } 1910 \\
\text { stets Brust; } 3-4 \text { stünd- } \\
\text { lich, } 120-130 \text { g pro } \\
\text { Mahlzeit } \\
\text { Teediät } \\
\text { Abstillung }\end{array}$ & $\begin{array}{l}\text { Seit } 3 \text { Wochen Ge- } \\
\text { wichtsstilistand, in der } \\
\text { letzten Zeit } 2 \text { grüne, } \\
\text { unverdaute Stihle täg- } \\
\text { lich }\end{array}$ & $\begin{array}{l}\text { 10. VIII. } \\
1910\end{array}$ & 2,5 & $\begin{array}{l}\text { Diese Zahl ent- } \\
\text { spricht den von } \\
\text { Moll angegebe- } \\
\text { nen Werten bei } \\
\text { Brustdyspep- } \\
\text { sien }\end{array}$ \\
\hline & & $\begin{array}{l}\text { Vom 11. VIII. bis } \\
22 \text {. VIII. } 6 \times 130 \cdot(1: 1) \\
\text { mit } 1 \% \text { Hafermehl, } \\
\text { nachdem Drittelmileh } \\
\text { mit Hafermeh ver- } \\
\text { tragen worden ist }\end{array}$ & $\begin{array}{l}\text { Bis zum 13. VIII. noch } \\
\text { schleimig dyspeptische } \\
\text { Stiihle; vom 14. bis } \\
22 \text {. 1 bis } 2 \text { homogene } \\
\text { Mehlstuhe; das Kind } \\
\text { befindet sich wohl bit } \\
\text { täglicher durchschnitt. } \\
\text { licher Zunahme um } \\
\quad 21,8 \mathrm{~g}\end{array}$ & 20. VIII. & 15,0 & $\begin{array}{l}\text { Die Phosphat- } \\
\text { zahl in einer } \\
\text { gesunden } \\
\text { Me blperiode } \\
\text { ist viel höher als } \\
\text { die in einer dys- } \\
\text { peptischen } \\
\text { Brustperiode }\end{array}$ \\
\hline \multirow[t]{3}{*}{31} & \multirow{3}{*}{$\begin{array}{l}\text { Sophie } \\
\text { Nikolajevir } \\
6 \text { Monate } \\
\text { Zwillings- } \\
\text { schwester } \\
\text { von } 30 \\
6 \text { Monate alt }\end{array}$} & $\begin{array}{c}\text { Ebentalls bis zum } \\
\text { 10. NIII. } 1910 \text { Brust } \\
\text { Teediat } \\
\text { tbstillung }\end{array}$ & $\begin{array}{l}\text { Seit der letzten Zeit } \\
\text { Gewichtsstillstand,täg- } \\
\text { lich } 2 \text { bis } 3 \text { dyspep- } \\
\text { tische Stuhle }\end{array}$ & 10. YI. & 7,05 & $\begin{array}{l}\text { Hohe Phos- } \\
\text { phatzahl ent- } \\
\text { sprechend der } \\
\text { Brustdyspepsie }\end{array}$ \\
\hline & & 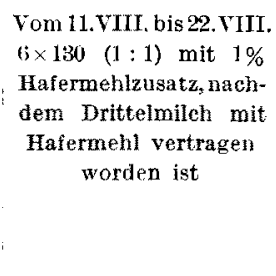 & $\begin{array}{l}\text { Bis zum 12. VIII. noch } \\
2 \text { bis } 3 \text { schleimig dys- } \\
\text { peptische,grüe Stuhhle, } \\
\text { seit } 13 \text {. VII, bomo- } \\
\text { gene Stüle. Tägliche } \\
\text { Zunahme um } 9,8 \text { g; das } \\
\text { Kind befindet sich wohl }\end{array}$ & 20. VIII. & 17,5 & $\begin{array}{l}\text { Die Phosphat- } \\
\text { zahl der ge* } \\
\text { sundenMehl- } \\
\text { periode ist viel } \\
\text { höher als die in } \\
\text { der voraluge- } \\
\text { gangenen dys- } \\
\text { peptischen } \\
\text { Brustperiode }\end{array}$ \\
\hline & & $\begin{array}{c}\text { Yom } 5.1 \mathrm{x} \text { bis } 2.1 \mathrm{~T} \text {. } \\
6 \times 130(1: 1) \text { bis } \mathrm{b} \times 1+0 \\
(1: 1) \text { mit } 3 \% \text { Mellins- } \\
\text { rahrung }\end{array}$ & $\begin{array}{l}\text { Vom } 8 . \text { IX. bis } 14 . \text { IX. } \\
\text { eine Pneumonie mit } \\
\text { einer Temperatur bis } \\
40^{\circ} \text {; die Verdaung } \\
\text { nicht wesentlich alte- } \\
\text { riert, wohl sehlechtere } \\
\text { Stihle, doch keine } \\
\text { starken Abnahmen }\end{array}$ & 13. IX. & 44,5 & $\begin{array}{l}\text { Diese hohe Zahl } \\
\text { entspricht einer } \\
\text { parenteralen } \\
\text { Störung (Pneu- } \\
\text { monie) }\end{array}$ \\
\hline
\end{tabular}




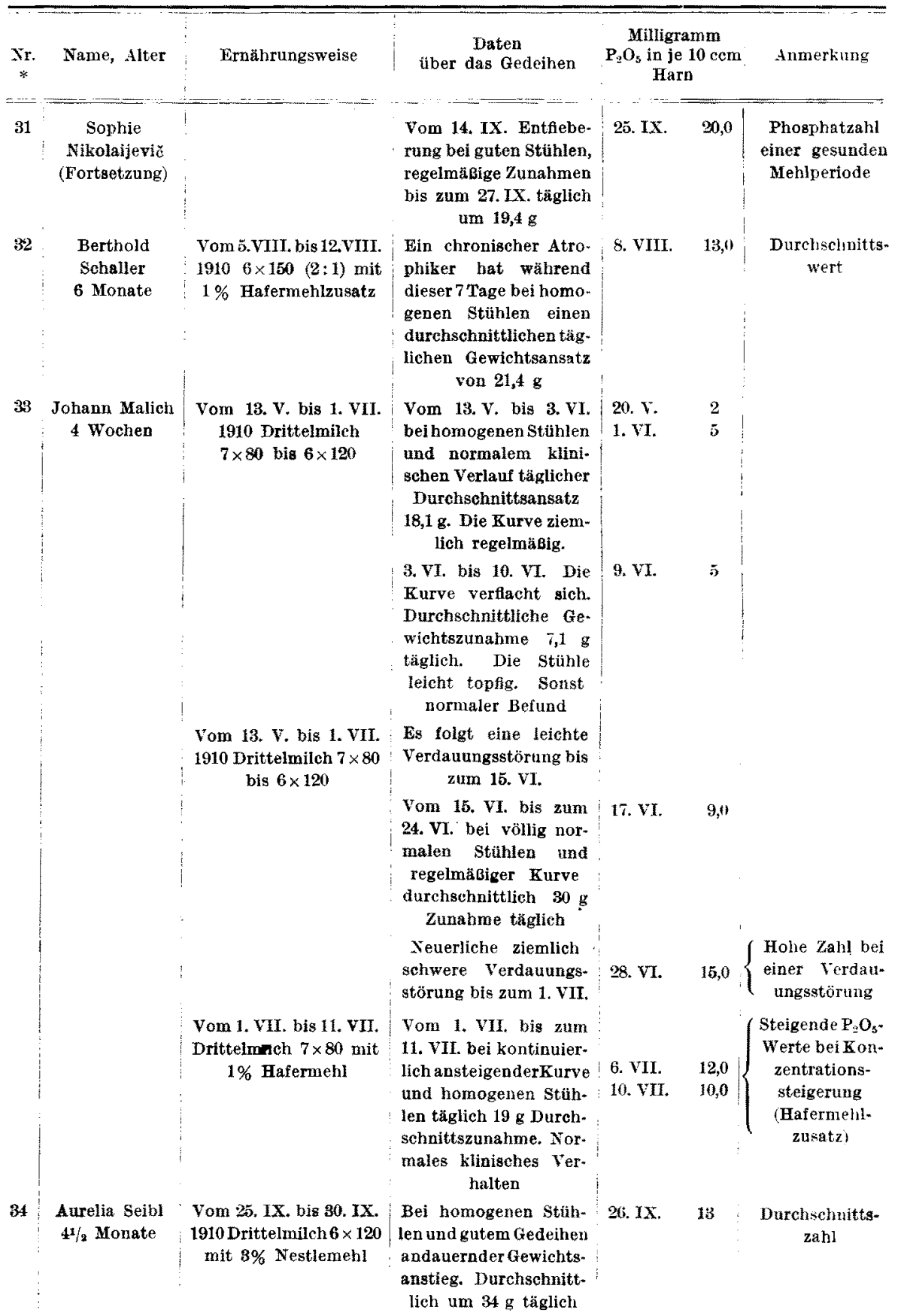




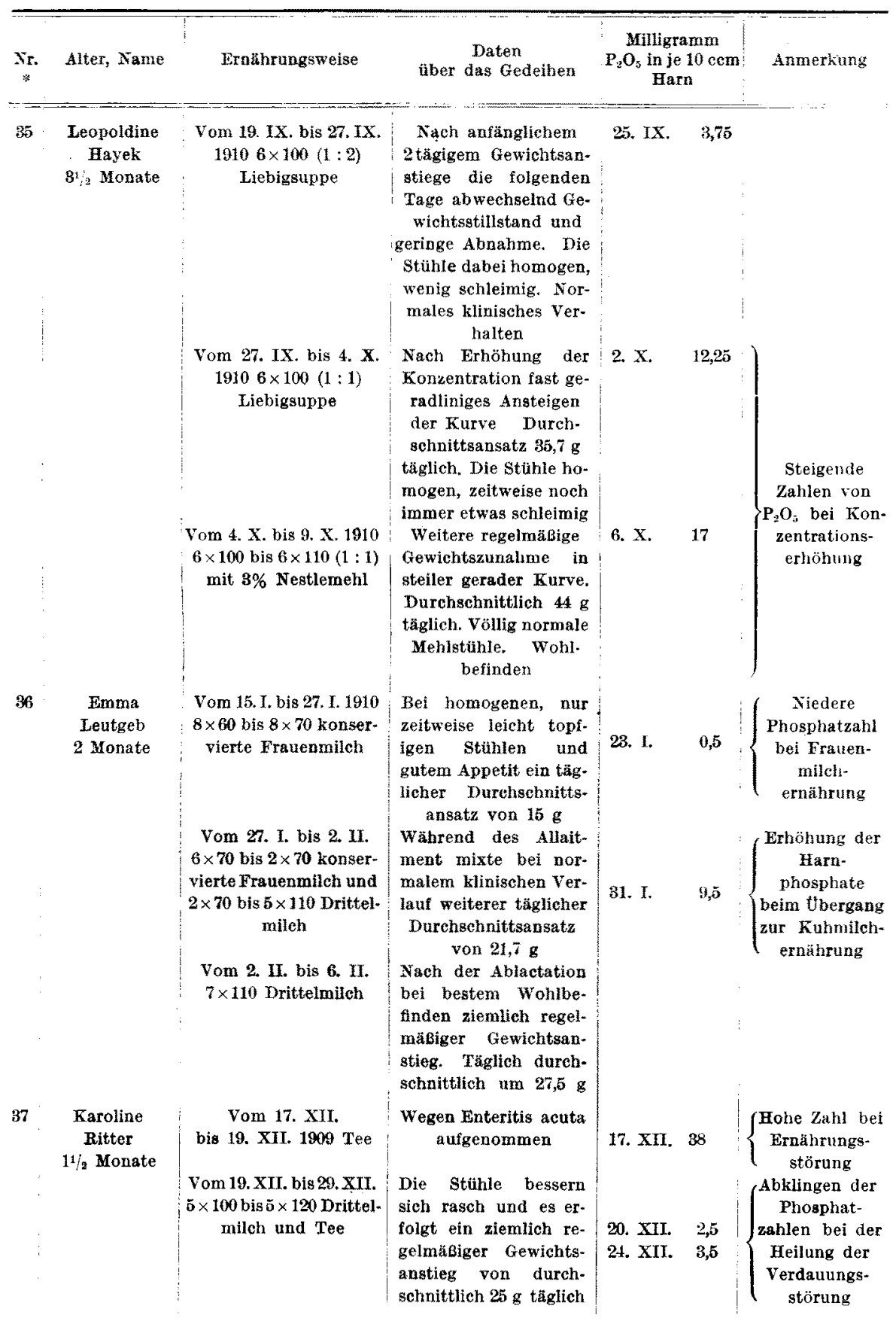




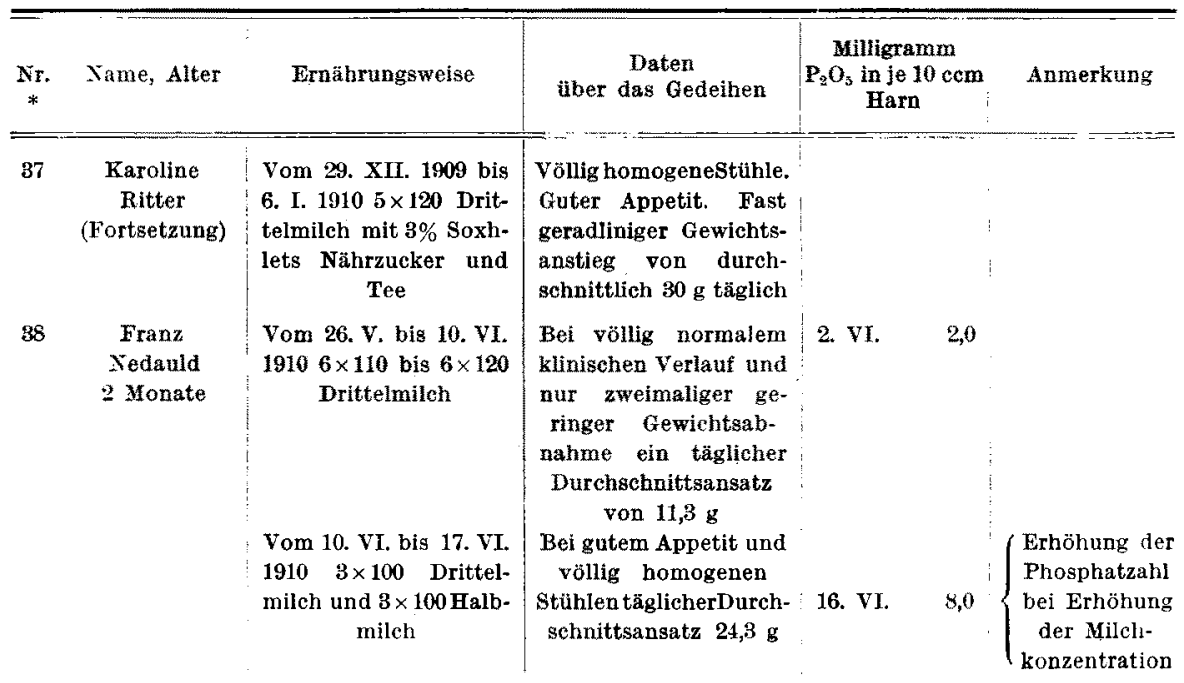

II.

Um gewisse in die Augen fallende Unregelmäßigkeiten bei der Phosphatbestimmung im Harne zu erklären, und mit den übrigen Befunden in Einklang zu bringen, war es nötig, eigens dahin gerichtete Cntersuchungen anzustellen. Von Mayerhofer ${ }^{1}$ ) wurde seinerzeit darauf aufmerksam gemacht, daß bei manchen Fällen von Magendarmstörungen die erste nach längerer Teediät gereichte Nahrung (auch Frauenmịlch) den Hungercharakter des Harnes sofort und vorübergehend verändert (Erhöhung der Permanganatzahl, eventuell auch Erhöhung der Phosphatzahl), so daß es in den entsprechenden Kurven den Anschein gewinnt, als ob durch die erste Nahrung alimentäre Zacken entstünden. Bei der Beurteilung der nun folgenden Versuche gewannen wir die Überzeugung, daß auch bei Veränderungen in der künstlichen Nahrung (Kohlehydratzufütterung) die Harnphosphate zackig ansteigen, in Analogie zu den früher von Mayerhofer gemachten Beobachtungen bei der ersten Nahrungsdarreichung nach Hungerdiät.

In den nun folgenden Versuchen gingen wir so vor, daB wir in je $10 \mathrm{ccm}$ Harn aus der 24 stündigen Menge- respektive aus einer möglichst großen Teilportion - die ausgeschiedene Menge der anorganischen Phosphate bestimmten und zwar derart, daß wir während 2-3 Wochen in Intervallen von 2-3 Tagen die Bestimmungen vormahmen. Dabei

1) E. Mayerhofer, Chemische Teilerscheinungen im Harne gesunder und kranker Säuglinge. Zeitschr. f. Kinderheilk. 1, 487. 1911. 
hat es sich nun gezeigt, daß es nicht gleichgültig war, an welchem Tage nach Beginn der Kohlehydratzufütterung wir die ausgeschiedene Phosphatmenge bestimmten, ein Ergebnis, das uns wichtig erscheint, weil durch diese Erkenntnis einige UnregelmäBigkeiten in der Phosphatausscheidung im Harne gesunder künstlich genährter Säuglinge bei Kohlehydratzufuhr teilweise erklärt werden können. Gleichzeitig wurde die in $10 \mathrm{~cm}$ Harn ausgeschiedene Stickstoff- (K jeldal) und Kochsalzmenge bestimmt. Zur Beobachtung gelangten nur Fälle, die in der Untersuchungsperiode magendarmgesund waren.

Wir lassen nun im folgenden einige uns charakteristisch erscheinende Fílle folgen $\left.{ }^{1}\right)$ :

\section{A. Künstlich genährte Sänglinge.}

Fall 39. Otto Krause, 4 Monate alt. Ein magendarmgesunder Säugling, bekommt als Nahrung $6 \times 100 \mathrm{~cm}$ halbverdünnte Kuhmilch. Wir bestimmten in je $10 \mathrm{ccm}$ der 24 stündigen Harnmenge die ausgeschiedene $\mathrm{P}_{2} \mathrm{O}_{5}$-Menge. Diese betrug in $10 \mathrm{ccm}$ Harn 7,5 mg, die gleichzeitig bestimmte Stickstoffmenge (K jeld a l) in $10 \mathrm{com} 24 \mathrm{mg}$, die CINa-Menge $20 \mathrm{mg}$. - Nach Zufütterung eines Kohlehydrates (Keller-Malzsuppe) steigt innerhalb der ersten Woche die im Harne ausgeschiedene $\mathrm{P}_{2} \mathrm{O}_{5}$-Menge von $7,5 \mathrm{mg}$ auf $30 \mathrm{mg}$, sinkt in den nächsten 2 Tagen auf $11,5 \mathrm{mg}$ herab, um sich auf diesem Niveau zu erhalten. Die gleichzeitig bestimmten Stickstoff- und Chlormengen bleiben von der Nahrungsänderung unbeeinflußt.

Fall 40. Ernst Kral, $2_{2}^{1}$ Monate alt. Ein magendarmgesunder Säugling bekommt als Nahrung $4 \times 100 \mathrm{~cm}$ halbverdünnte Kuhmilch und $2 \times 80 \mathrm{~cm}$ Frauenmilch. Die in $10 \mathrm{cem}$ Harn ausgeschiedene $\mathrm{P}_{2} \mathrm{O}_{5}$-Menge beträgt $4 \mathrm{mg}$. Die Stickstoffmenge $13 \mathrm{mg}$, die Kochsalzmenge $12 \mathrm{mg}$. Nach Zufütterung von Kohlehydrat (Keller-Malzsuppe) steigt bei Gewichtszunahme die Menge der ausgeschiedenen $\mathrm{P}_{2} \mathrm{O}_{5}$ in der ersten Woche von $4 \mathrm{mg}$ auf $27 \mathrm{mg} \mathrm{P}_{2} \mathrm{O}_{5}$, sinkt nach weiteren 5 Tagen auf $12 \mathrm{mg}$ herab, um auf diesem Niveau zu verharren. Während der Versuchsperiode blieb die ausgeschiedene Stickstoff- und Cl-Menge unverändert.

Fall 41. Eduard von Mayer, 4 Monate alt. Fin magendarmgesundes Kind, bekommt $3 \times 100 \mathrm{~g}$ Frauenmilch und $3 \times 100 \mathrm{~g}$ Finkelsteinsche Eiweißmilch. Die in $10 \mathrm{ccm}$ Harn ausgeschiedene $\mathrm{P}_{2} \mathrm{O}_{5}$-Menge beträgt $3,5 \mathrm{mg}$. Die in $10 \mathrm{ccm}$ ausgeschiedene Stickstoffmenge beträgt $15 \mathrm{mg}$, die Kochsalzmenge $12 \mathrm{mg}$. Nach Zufütterung ron Nährzucker steigt die Menge der im Harne ausgeschiedenen Phosphatmenge in der ersten Woche von $3,5 \mathrm{mg}$ anf $15,4 \mathrm{mg}$ und sinkt nach 4 weiteren Tagen auf $9 \mathrm{mg}$ herab. Die ausgeschiedenen Chlornatrium- und Stickstoffmengen ändern sich nicht. Das Kind nimmt während des Versuches an Gewicht zu.

Fall 42. Anton Reischer, 4 Monate alt. Ein magendarmgesunder Säugling, bekommt als Nahrung $6 \times 140 \mathrm{~cm}$ halbverdünnte Kuhmilch mit $4 \%$ Soxhlets Nährzucker. Die in $10 \mathrm{ccm}$ Harn ausgeschiedene Phosphatmenge beträgt $3 \mathrm{mg}$,

1) In der Tabelle auf $\mathbf{S . 4 4 / 4 5}$ erscheinen diese Fälle kurz zusammengestellt. 


\begin{tabular}{|c|c|c|c|c|c|c|c|}
\hline $\begin{array}{l}\text { Fall-Nr. } \\
\text { Tame }\end{array}$ & Alter & $\begin{array}{c}\text { Anfingliohe } \\
\text { Nahrung } \\
\text { in ccm }\end{array}$ & $\begin{array}{l}\text { Phosphate, } \\
\text { N (Kjeldal), } \\
\text { ClNa inMilli- } \\
\text { grammen in } \\
\text { je } 10 \text { ecm } \\
\text { Harn }\end{array}$ & $\begin{array}{l}\text { Nahrungs- } \\
\text { wechsel }\end{array}$ & $\begin{array}{l}\text { Phospbate, } \\
\text { N (Kjeldal), } \\
\text { CINa in Milli- } \\
\text { grammen in } \\
\text { je } 10 \mathrm{ccm} \\
\text { Harn }\end{array}$ & $\begin{array}{c}\text { Daner } \\
\text { des } \\
\text { Ver } \\
\text { suches }\end{array}$ & $\begin{array}{l}\text { Beschrei- } \\
\text { bung der } \\
\text { Harn- } \\
\text { portionen }\end{array}$ \\
\hline $\begin{array}{c}\text { Nr. } 39 . \\
\text { Otto Krause }\end{array}$ & $\begin{array}{c}4 \\
\text { Ion. }\end{array}$ & $\begin{array}{c}6 \times 100 \\
\text { Halbmilch }\end{array}$ & $\begin{array}{l}\text { 1. Tag: } \\
\mathrm{P}_{2} \mathrm{O}_{5}=7,5 \\
\mathrm{ClNa}=20,0 \\
\mathrm{~N}=24 \\
7 . \mathrm{Tag} \\
\mathrm{P}_{2} \mathrm{O}_{3}=9,5 \\
\mathrm{ClNa}=20,3 \\
\mathrm{~N}=24\end{array}$ & $\begin{array}{l}6 \times 100 \\
\text { Kellers Malz- } \\
\text { suppe mit } \\
\text { Halbmilch am } \\
7 \text { Tag }\end{array}$ & $\begin{array}{l}14 . \mathrm{Tag}: \\
\mathrm{P}_{2} \mathrm{O}_{3}=30,0 \\
\mathrm{ClNa}=20,0 \\
\mathrm{~N}=24,4 \\
21 . \mathrm{Tag}: \\
\mathrm{P}_{2} \mathrm{O}_{5}=11,5 \\
\mathrm{CINa}=20,2 \\
\mathrm{~N}=24,1 \\
32 . \mathrm{Tag}: \\
\mathrm{P}_{2} \mathrm{O}_{5}=11,5 \\
\mathrm{CLa}=20,0 \\
\mathrm{~N}=24,0\end{array}$ & 32 Tage & $\begin{array}{l}24 \text { stuin: } \\
\text { dige } \\
\text { Menge }\end{array}$ \\
\hline $\begin{array}{l}\text { Nr. } 40 . \\
\text { Ernst Kral }\end{array}$ & $\begin{array}{l}2^{21} \\
\text { Mon. }\end{array}$ & $\mid \begin{array}{c}4 \times 100 \\
\text { Halbmilch, } \\
2 \times 80 \\
\text { Frauenmileh }\end{array}$ & $\begin{array}{l}\text { 1. Tag: } \\
\mathrm{P}_{2} \mathrm{O}_{5}=4,0 \\
\mathrm{ClNa}=12,0 \\
\mathrm{~N}=13,0 \\
7 . \mathrm{Tag}: \\
\mathrm{P}_{2} \mathrm{O}_{5}=4,5 \\
\mathrm{ClNa}=12,0 \\
\mathrm{~N}=18,1\end{array}$ & $\begin{array}{c}3 \times 60 \\
\text { Frauenmilch } \\
\text { und } 3 \times 60 \\
\text { Kellers Malz* } \\
\text { suppe }(5 \%) \text { mit } \\
\text { Halbmilch am } \\
7 . \text { Tag }\end{array}$ & $\begin{array}{c}\text { 14. Tag: } \\
\mathrm{P}_{2} \mathrm{O}_{5}=27,0 \\
\mathrm{CWNa}=12,2 \\
\mathrm{~N}=13,0 \\
19 . \mathrm{Tag} \\
\mathrm{P}_{5} \mathrm{O}_{5}=12,0 \\
\mathrm{ClNa}=12,0 \\
\mathrm{~N}=13,5 \\
27 . \mathrm{Tag}: \\
\mathrm{P}_{2} \mathrm{O}_{5}=12,0 \\
\mathrm{CINa}=12,5 \\
\mathrm{~N}=13,0\end{array}$ & 27 Tage! & $\begin{array}{l}24 \text { stün- } \\
\text { dige } \\
\text { Menge }\end{array}$ \\
\hline $\begin{array}{l}\text { Nr. } 41 . \\
\text { Eduard } \\
\text { von Yayer }\end{array}$ & Mon. & $\begin{array}{c}3 \times 100 \\
\text { Frauen- } \\
\text { milch, } \\
3 \times 100 \\
\text { Finkelstein- } \\
\text { sche Eiweilo- } \\
\text { milch }\end{array}$ & $\begin{array}{c}\text { 1. Tag: } \\
\mathrm{P}_{2} \mathrm{O}_{5}=3,5 \\
\mathrm{CINa}=12,2 \\
\mathrm{~N}=15,0 \\
6 . \mathrm{Tag}: \\
\mathrm{P}_{2} \mathrm{O}_{5}=3,5 \\
\mathrm{CINa}=12,0 \\
\mathrm{~N}=15,0\end{array}$ & $\begin{array}{c}3 \times 100 \\
\text { Frauenmilch, } \\
3 \times 100 \\
\text { Finkelsteinsche } \\
\text { EiweiBmilch } \\
\text { mit } 12 \mathrm{~g} \\
\text { Soxhlets } \\
\text { Nahrzacker am } \\
6 . \text { Tag }\end{array}$ & $\begin{array}{c}\text { 11. Tag: } \\
\mathrm{P}_{2} \mathrm{O}_{5}=15,4 \\
\mathrm{CWTa}=12,1 \\
\mathrm{~N}=15,2 \\
10 . \mathrm{Tag}: \\
\mathrm{P}_{2} \mathrm{O}_{5}=9,0 \\
\mathrm{CN}=12,05 \\
\mathrm{~N}=15,02 \\
21 . \mathrm{Tag}: \\
\mathrm{P}_{2, \mathrm{O}_{5}}=9,0 \\
\mathrm{CNa}=12,05 \\
\mathrm{~N} .=15,01\end{array}$ & 21 Tage & $\begin{array}{l}2+\text { stiu- } \\
\text { dige } \\
\text { Menge }\end{array}$ \\
\hline $\begin{array}{c}\text { Nr. } 42 \\
\text { Anton } \\
\text { Reischer }\end{array}$ & $\stackrel{4}{4}$ & $\begin{array}{c}6 \times 140 \\
\text { Halbmilch } \\
\text { mit } 4 \% \\
\text { Soxhlets } \\
\text { Nährucker }\end{array}$ & $\begin{array}{l}\text { 1. Tag: } \\
\mathrm{P}_{2} \mathrm{O}_{5}=\mathbf{3 , 0} \\
\mathrm{CINa}=18,01 \\
\mathrm{X}=24,02 \\
\text { t. Tag: } \\
\mathrm{P}_{2} \mathrm{O}_{5}=2,0 \\
\mathrm{CINa}=18,0 \\
\mathrm{X \quad}=24,02\end{array}$ & $\begin{array}{c}6 \times 140 \\
\text { Halbmilch mit } \\
4 \% \text { Soxhleths } \\
\text { Nahrzucker } \\
\text { und } 2 \% \\
\text { Weizenmehl }\end{array}$ & 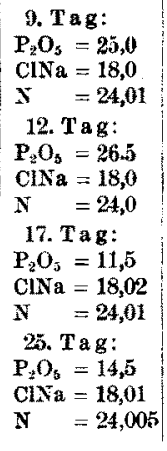 & 25 Tage & $\begin{array}{l}24 \text { stinn- } \\
\text { dige } \\
\text { Menge }\end{array}$ \\
\hline
\end{tabular}




\begin{tabular}{|c|c|c|c|c|c|c|c|}
\hline $\begin{array}{c}\text { Fall Sr. } \\
\text { Name }\end{array}$ & Alter & $\begin{array}{l}\text { Anfängliche } \\
\text { Nahrung } \\
\text { in com }\end{array}$ & $\begin{array}{l}\text { Phosphate, } \\
\text { N(Kjeldal)' } \\
\text { CLNa in Milli- } \\
\text { grammen in } \\
\text { je } 10 \mathrm{ccm} \\
\text { Harn }\end{array}$ & $\begin{array}{l}\text { Nahrungs" } \\
\text { wechsel }\end{array}$ & $\begin{array}{l}\text { Phosphate, } \\
\times \text { (Kjeldal), } \\
\text { ClNa in Milli- } \\
\text { grammen in } \\
\text { je } 10 \mathrm{ccm} \\
\text { Harn }\end{array}$ & $\begin{array}{c}\text { Dauer } \\
\text { des } \\
\text { Ver- } \\
\text { suches }\end{array}$ & $\begin{array}{l}\text { Beschrei- } \\
\text { bung der } \\
\text { Harn- } \\
\text { portionen }\end{array}$ \\
\hline $\begin{array}{l}\text { Nr. } 43 . \\
\text { I.lly Fried }\end{array}$ & $\begin{array}{c}3 \\
\text { Mon. }\end{array}$ & $\begin{array}{l}\text { Casein- } \\
\text { Iösung nach } \\
\text { Mayerhofer- } \\
\text { Holl mit } \\
6 \% \text { Xahr- } \\
\text { aucker }\end{array}$ & $\begin{array}{c}\text { 1. Tag: } \\
\mathrm{PO}_{3}=7,0 \\
\mathrm{ClNa}=0,81 \\
\mathrm{X}=0,902 \\
3 . \mathrm{Tag} \\
\mathrm{P}_{3}=6,0 \\
\mathrm{CH}=0,81 \\
\mathrm{X}=0,971\end{array}$ & $\begin{array}{c}\text { Caseinlosung } \\
\text { nach Mayer- } \\
\text { hofer-Holl nit } \\
6 \% \text { Nânrucker } \\
\text { und } 3 \% \text { Kufeke- } \\
\text { nehl }\end{array}$ & 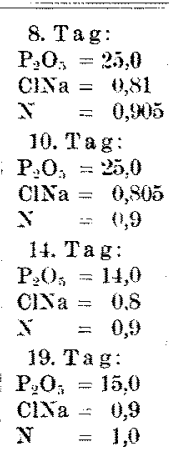 & 19 Tage & $\begin{array}{l}\text { Gröber } \\
\text { Ham- } \\
\text { portionen } \\
\text { ron ea. } \\
200 \mathrm{~cm}\end{array}$ \\
\hline $\begin{array}{l}\text { Xr. } 4 . \\
\text { Helene } \\
\text { schindler }\end{array}$ & $\begin{array}{c}4 \\
\text { Mon. }\end{array}$ & $\begin{array}{c}3 \times 120 \\
\text { Frauen- } \\
\text { milch u. } \\
3 \times 120 \\
\text { Halbmilch }\end{array}$ & $\begin{array}{c}\text { 1. Tag: } \\
\mathrm{P}_{2} \mathrm{O}_{5}=5,0 \\
\mathrm{CNa}=12,002 \\
\mathrm{~N}=13,001 \\
\text { o. Tag: } \\
\mathrm{P}_{5} \mathrm{O}_{3}=5,0 \\
\mathrm{CNNa}=12,05 \\
\mathrm{X}=13,002\end{array}$ & $\begin{array}{c}3 \times 120 \\
\text { Franenmilch, } \\
3 \times 120 \text { Halb- } \\
\text { milch mit } 3 \% \\
\text { Kufekemehl }\end{array}$ & $\begin{array}{c}10 . \mathrm{Ta}: \\
\mathrm{P}_{2} \mathrm{O}_{5}=1,0 \\
\mathrm{ClNa}=12,00 \mathrm{~B} \\
\mathrm{~N}=13,001 \\
15 . \mathrm{Tag}: \\
\mathrm{P}_{2} \mathrm{O}_{3}=10,0 \\
\mathrm{ClNa}=12,02 \\
\mathrm{~N}=13,0 \\
20 . \mathrm{Tag}: \\
\mathrm{P}_{2} \mathrm{O}_{-}=10,0 \\
\mathrm{ClNa}=1 \cdot 0 \\
\mathrm{~N}=13,0\end{array}$ & 20 Tage & $\begin{array}{l}\text { Gröbere } \\
\text { Harn- } \\
\text { portionen } \\
\text { von ca. } \\
230 \mathrm{ccm}\end{array}$ \\
\hline
\end{tabular}

die Chloridmenge $18 \mathrm{mg}$, die Stickstoffmenge $24 \mathrm{mg}$. Nach einem Zusatz ron $2 \%$ Weizenmehl zur Nahrung steigt bei ansteigendem Gewichte die im Harne ausgeschiedene Menge in den ersten 6 Tagen ron $3 \mathrm{mg}$ auf 25 und 26,5 $\mathrm{mg}$, sank nach weiteren 7 Tagen auf $11,5 \mathrm{mg}$ herab, auf welchem Niveau sie beiläufig blieb. Die Stickstoff- und Chlornatriumausscheidung wurde von der Nahrungsänderung nicht beeinflußt.

Fall 43. Lilly Fried, 3 Monate alt. Ein magendarmgesundes Kind, bekommt als Nahrung alkalische Caseinlösung (Ma yerhofer - Moll) mit 6\% Nährzucker. Die in $10 \mathrm{~cm}$ einer $200 \mathrm{ccm}$ betragenden Harnportion bestimmten ausgeschiedenen Phosphate betragen um 6 und $7 \mathrm{mg}$. Die Chloridmenge ist $6,8 \mathrm{mg}$, die Stickstoffmenge $0,9 \mathrm{mg}$. Xach einem Zusatz von $3 \% \mathrm{~K} \mathrm{ufekemehl}$ steigt innerhalb 5 Tagen die im Hame ausgeschiedene Phosphatmenge auf $25 \mathrm{mg}$, und sinkt in der nächsten Woche auf $14 \mathrm{mg}$ herab, auf welchem Niveau sich die Zahl erhält. Die Stickstoff- und Chlorausscheidungen bleiben unbeeinfluBt. Das Kind nimmt während der Versuche an Gewicht zu.

Fall 44. Helene Schindler, 4 Monate alt. Ein magendarmgesunder Säugling, bekommt als Xahrung $3 \times 120 \mathrm{~cm}$ Fravenmileb $+3 \times 120 \mathrm{~cm}$ halbverdünnte Kuhmilch. Die Menge der im Harn (10 ccm) ausgeschiedenen Phosphate beträgt $5 \mathrm{mg}$, Chlornatrium $12 \mathrm{mg}$, Stickstoff $13 \mathrm{mg}$. Nach Zufüterung von $3 \% \mathrm{Ku}$ feke steigt in der ersten Woche die Menge der anorganischen Phosphate auf $25 \mathrm{mg}$, 
sinkt in der nächsten Woche auf $10 \mathrm{mg}$, um auf diesem Niveau zu verharren. Die Stickstoff- und Chlornatriumausscheidung bleibt unbeeinflußt. Das Kind nimmt während der Versuche an Gewicht zu.

Zur besseren Illustration dieser Verhältnisse möge noch eine Kurve gebracht werden. Es genügt Fall 42, denn alle übrigen folgen mit nur unwesentlichen Verschiedenheiten diesem gezeichneten Paradigma (Fig. 6).

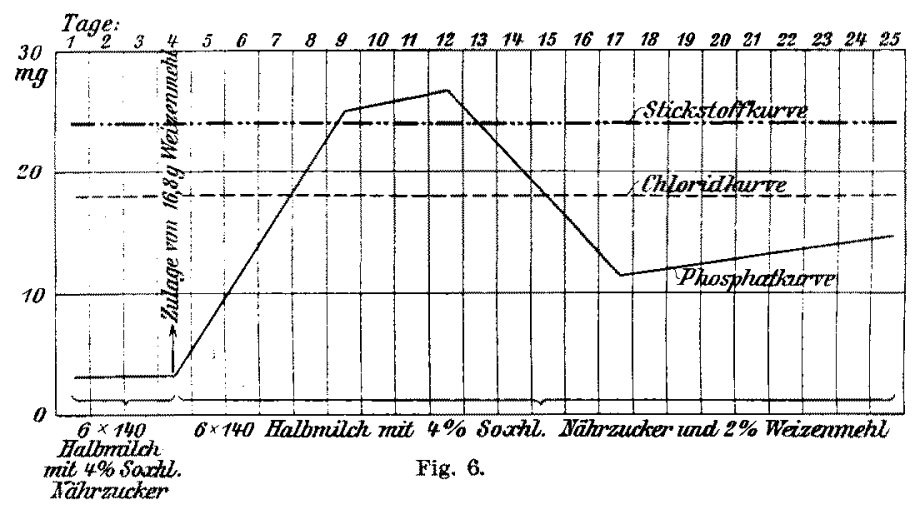

Die vorstehende Tabelle und Kurve zeigt uns, daß bei Kohlehydratzufütterung - und zwar wurden sowohl verschiedene Mehle wie auch Soxhlets Nährzucker in die Versuche mit einbezogen - in den ersten Tagen im Harne magendarmgesunder künstlich ernährter Säuglinge ein bedeutender Anstieg von im Harn ausgeschiedenem anorganischen Phosphor, zu konstatieren ist, welche Mehrausscheidung nach einigen Tagen wieder abnimmt, um auf einem meist höheren Niveau als vor Beginn der Kohlehydratzufütterung zu verharren. Dabei sei nochmals betont, daß diese Kinder während der Versuchsperiode an Gewicht zunahmen und sowohl die Stickstoff- wie die Chlorausscheidung unbeeinfluBt blieb. Nach Feststellung dieser Tatsachen war es uns interessant, nachzusehen, ob natürlich genährte Säuglinge bei Kohlehydratzufütterung ein ähnliches Verhalten zeigen. $\mathrm{Zu}$ diesem Zweck gaben wir zur Frauenmilchnahrung solcher Säuglinge, die in der Versuchsperiode magendarmgesund waren, Kohlehydrate in Wasser als Beinahrung.

Im Nachstehenden seien kurz 5 solcher Fälle beschrieben.

Fall 45. Pia Clementine, 21/2 Monate alt. Ein magendarmgesunder Säugling, bekommt als Nahrung $6 \times 80 \mathrm{ccm}$ Frauenmilch. Bei guter Gewichtszunahme sind im Harne nach der oben beschriebenen Weise keine anorganischen Phosphate nachweisbar. Nach Zufütterung von $2 \times 3 \mathrm{~g}$ Kufeke in je $100 \mathrm{ccm}$ Wasser bleiben die ausgeschiedenen Phosphate $=0$.

Fall 46. Leopold Klatzmayer, 3 Monate alt. Ein magendarmgesunder Säugling, bekommt als Nahrung $6 \times 90 \mathrm{~g}$ Frauenmilch. Bei guter Gewichtszunahme 
sind im Hame nach oben beschriebener Weise keine anorganischen Phosphate nachweisbar. Nach Zufütterung von Keller-Malzsuppe $(3 \times 5 \mathrm{~g}$ Kellers Malz in je $90 \mathrm{ccm}$ Wasser und $3 \times 90$ Frauenmilch) bleiben die ausgeschiedenen Phosphate $=0$.

Fall 47. Franz Reichenauer, 10 Wochen alt. Ein magendarmgesunder Säug. ling bei reiner Brustnahrung $(6 \times 90 \mathrm{~cm})$. Bei guter Gerwichtszunahme sind im Harne nach oben beschriebener Weise keine anorganischen Phosphate nachweisbar. Nach Zufütterung von $2 \times 1003$ proz. Kufekewassers mit Saccharin bleibt die ausgeschiedene Phosphatmenge $=0$.

Fall 48. Leopold Baumann, 12 Wochen alt; ein magendarmgesunder Säugling, bekommt als Nahrung $5 \times 150 \mathrm{~cm}$ Frauenmilch. Bei guter Gewichtszunahme sind nach oben beschriebener Weise keine anorganischen Phosphate im Harne nachweisbar. Nach Zufütterung von $3 \times 3 \mathrm{~g}$ Kufeke in je $80 \mathrm{~g}$ Wasser bleiben die Phosphate $=0$.

Fall 49. Hermine Flach, 2 Monate alt. Ein magendarmgesunder Säugling, bekommt als Nahrung $5 \times 150 \mathrm{~cm}$ Franenmilch. Bei guter Gewichtszunahme sind nach oben beschriebener Weise keine anorganischen Phosphate im Harne nachweisbar. Nach Zufütterung von einer Mahlzeit $100 \mathrm{cem}$ Kufekewassers dureb 2 Tage bleiben die ausgeschiedenen Phosphate $=0$.

Wie diese Fälle zeigen, verhalten sich die lediglich von Frauenmilch ernährten, magendarmgesunden Säuglinge resistenter gegenüber der $\mathrm{P}_{2} \mathrm{O}_{5}$-Ausscheidung im Harne nach Kohlehydratzufütterung als künstlich ernährte. Sie scheiden nämlich trotz Kohlehydratzufütterung keine - nach der titrimetrischen Untersuchungsmethode nachweisbare anorganische Phosphate im Harne aus.

Fall 50. Da wir daran dachten, daß es sich bei der erhöhten Phosphatausfuhr ähnlich wie bei der Acydose um eine Ansäuerung des Organismus handle, haben wir einem magendarmgesunden Säugling $3 \times 0,1$ Benzoesäure verfüttert und den Harn in analoger Weise anf seinen $\mathrm{P}_{2} \mathrm{O}_{5}$-Gehalt untersucht. Es hat sich dabei gezeigt, daß magendarmgesunde, künstlich emährte Kinder bei Gewiohtszunahme nach Verabreichung von Benzoesäure einen Anstieg der $\mathrm{P}_{2} \mathrm{O}_{5}$ - Werte im Harne zeigen, der nach einigen Tagen sinkt, um auf einem immerhin noch gegen den Anfang erhöhten Niveau zu bleiben. Die Phosphatausseheidung betrug vor der Benzoesäuredarreichung $10 \mathrm{mg}$ in $10 \mathrm{~cm}$ Ham, stieg nach $3 \times 0,1$ Benzoesäure auf $20 \mathrm{mg}$ und sank am 9. Tage des Versuches auf $12 \mathrm{mg}$ herab.

Koep pe ${ }^{1}$ ) suchte gleichfalls durch Untersuchung von Einzelportionen des Harns verschiedener gesunder Flaschenkinder nach Gesetzmäßigkeiten. Schọ an seinem kleinen Material fand er eine Abhängig keit der Phosphatausscheidung von der Art der Nahrung und bei derselben Nahrung eine scheinbare Abhängigkeit von der Nahrungsmenge. Diese allgemeine Beobachtung erscheint durch unsere Massenbeobachtungen bestätigt und ist nach unseren Erfahrungen noch vielfach in

1) H. Koeppe, Studien zum Mineralstoffwechsel. Jahrb. f. Kinderheilk. 73. 0.1911. 
Einzelheiten erweitert worden. - Auch wir machten die Beobachtung; daß unter den Kohlehydraten das Hafermehl eine Ausnahmsstellung insofern einnimmt, als die Phosphatausscheidungen bei Hafermehlmilchmischungen geringer erschienen. Der Beobachtung Koeppes, daß eine Zugabe von $1 \mathrm{~g}$ Kochsalz zur Nahrung eine erhebliche Steigerung der $\mathrm{P}_{2} \mathrm{O}_{5}$-Ausfuhr zur Folge hat, wollen wir unsere Beobachtung zur Seite stellen, daß eine ähnliche Steigerung der $\mathrm{P}_{2} \mathrm{O}_{5}$-Ausfuhr auch nach $3 \times 0,1$ Benzoesäure pro die eintrat. Wahrscheinlich treten ähnliche Phosphatausschwemmungen auch noch nach anderen Stoffen ein, weshalb wir die Frage der Ätiologie einstweilen unbeantwortet lassen wollen. Wir dachten bei unseren Versuchen an einen Zusammenhang der Acidose mit einer vermehrten Phosphatausscheidung; doch liegt die endgültige Entscheidung über diese Frage auBerhalb des Rahmens dieser rein klinischen Arbeit.

Hierbei sei noch erwähnt, dab Brustkinder auch bei Benzoesäurezufütterung in ihrem Phosphatstoffwechsel unbeeinflußt bleiben.

Resumee: Fassen wir unsere Erfahrungen an den mitgeteilten Fällen kurz zusammen, so ergibt sich:

A. Die anorganischen Phosphate steigen im Harne des künstlich genährten Säuglings bei:

1. Verdauungsstörungen aus enteralen Ursachen,

2. Parenteralen Störungen,

3. bei Frhöhung der Konzentration der künstlichen Nahrung:

a) beim U̇bergang von der Teediät zu irgendeiner künstlichen Nahrung.

b) bei Verstärkung der Kuhmilchkonzentration,

c) bei Zuckerzusatz,

d) bei Mehlzusatz.

4. Beim L'bergang ron Frauenmileh zu einex Kuhmilch-oder Kuhmilchzucker- oder Kuhmilchmehlmischung.

B. Die anorganischen Phosphate sinken im Harne des künstlich genährten S̈̈uglings bei:

1. Heilung einer Magendarmstörung,

2. Heilung einer parenteralen Störung,

3. Verminderung dex Konzentration der künstlichen Nahrung,

4. Eintritt des Hungerzustandes.

C. Man findet die angeführten Sohwankungen derausgeschiedenen anorganischen Phosphate beider überwiegenden Anzahl von Fällen nichtallein in der 24 stündigen Harn- 
menge, sondern auch in einzelnen gröBeren oder kleineren Teilportionen.

D. Bei künstlich ernährten, magendarmgesunden Säuglingen steigt in den ersten Tagen nach Kohlehydratzufütte. rung die Menge der im Harn ausgeschiedenen Phosphatean, sinktabernach $1-2$ Wochen trotz gleichbleibender Nahrung wieder ab.

1. Bei Mehlzufuhr ist im allgemeinen der Wert der ausgeschiedenen anorganischen Harmphosphate höher als bei Zuckerzufuhr; die geringste Erhöhung scheint Hafermehl zu verursachen.

2. Die Stickstoff- und Chlorausscheidung im Hame bleibt hierbei unbeeinflußt.

3. Nach Verfütterung von Benzoesäure steigt die Phosphatausfuhr im Harne künstlich genährter Säuglinge.

E. Gesunde Brustkinder zeigen weder bei mäBiger Kohlehydratzufütterung noch bei Verabreichung von Benzoesäure eine Vermehrung ihrer im Harn ausgeschiedenen Phosphatmenge.

F. In Ergänzung zu den in der Literatur vorliegenden Befunden kommen wir zu dem Schlusse, daß die Phosphattitration im Harne künstlich genährter Säuglinge nur dann klinisch verwertet werden kamn, wemn alle angefühten Punkte berücksichtigt werden.

G. Nach unserer Meinung befindet sich der Stoffwechsel des künstlich genährten Kindes fortwährend in einem labilen und nicht ganz normalen Zustande; es besteht regelmäBig eine geringe Phosphaturie. Wenn die Nahrung vermehrt, in ihrer Konzentration oder in ihrem Gehalte an Kohlehydraten erhöht wird, so tritt eine Stoffwechselstörung in Form der Phosphaturie stark zutage. Der Organismus gewöhnt sich aber meistens bald an die neue Nahrung, worauf wieder ein Rückgang der Phosphaturie auf geringere Werte erfolgt. - In ähnlicher Weise entsteht eine Phosphaturie, wenn durch parenterale Ursachen die Assimilationskraft des Organismus herabgesetzt ist.

H. Die normale Brustnahrung wird vom gesunden Brustkinde ohne jede Phosphaturie verdaut. Jede Phosphaturie bedeutet hier eine Verdauungsstörung (Moll).

I. Der klinische Wert der Phosphattitration in Harn des Flaschenkindes ist ein sehr beschränkter.

Dr. Gisa Kaminer, Wien IX/2 Lazarettgasse $\mathbf{1 4 .}$

Dr. phil. et med. Ernst Mayerhofer, Wien IX/2 Lazarettgasse 1t. 